\title{
Experimental Study of Active Vibration Control of Planar 3-ㄹRR Flexible Parallel Robots Mechanism
}

\author{
Qinghua Zhang, ${ }^{1}$ Xianmin Zhang, ${ }^{2}$ and Junyang Wei ${ }^{2}$ \\ ${ }^{1}$ Department of Mechatronics, Foshan University, Foshan, Guangdong 528000, China \\ ${ }^{2}$ Key Laboratory of Precision Equipment and Manufacturing Technology of Guangdong Province, Wushan Road, \\ Tianhe District, Guangzhou 510641, China \\ Correspondence should be addressed to Xianmin Zhang; zhangxm@scut.edu.cn
}

Received 21 April 2016; Revised 15 July 2016; Accepted 20 July 2016

Academic Editor: Francesco Ripamonti

Copyright (C) 2016 Qinghua Zhang et al. This is an open access article distributed under the Creative Commons Attribution License, which permits unrestricted use, distribution, and reproduction in any medium, provided the original work is properly cited.

\begin{abstract}
An active vibration control experiment of planar 3-RRR flexible parallel robots is implemented in this paper. Considering the direct and inverse piezoelectric effect of PZT material, a general motion equation is established. A strain rate feedback controller is designed based on the established general motion equation. Four control schemes are designed in this experiment: three passive flexible links are controlled at the same time, only passive flexible link 1 is controlled, only passive flexible link 2 is controlled, and only passive flexible link 3 is controlled. The experimental results show that only one flexible link controlled scheme suppresses elastic vibration and cannot suppress the elastic vibration of the other flexible links, whereas when three passive flexible links are controlled at the same time, they are able to effectively suppress the elastic vibration of all of the flexible links. In general, the experiment verifies that a strain rate feedback controller is able to effectively suppress the elastic vibration of the flexible links of plane 3-RRR flexible parallel robots.
\end{abstract}

\section{Introduction}

Recently, flexible parallel robots have been intensively studied because of their excellent performance. However, the elastic vibration problem of such robots is serious due to their inertial and driving forces. As a result, suppressing the unwanted elastic vibration is currently a very significant and challenging problem.

There are basically five design philosophies for suppressing unwanted elastic vibration that are classified into passive vibration control and active vibration control [1]. Passive vibration control means that unwanted vibration is suppressed by using advanced composite materials, optimizing the cross-sectional geometry of the elements, or using additional damping materials. Active vibration control means that unwanted vibration is suppressed by introducing a microprocessor-controlled actuator into the original mechanism or embedding some smart structures (actuators and sensors) into flexible elements. The surface-bonded lead zirconate titanate (PZT) sensor/actuator has been widely used for active vibration control of the flexible structure $[2,3]$.
PZT materials are able to dampen vibration and measure the vibration of distributed parameter systems because of their direct and inverse piezoelectric effect [4]. Photostrictive actuator as a new promising noncontact photoactuation technique was used for suppressing vibration of flexible structures [5].

During the past few decades, the question of active vibration control of flexible robots has been paid considerable attention, as indicated in survey papers [6-9]. Although there are many theoretical and experimental studies of active vibration control in space-based flexible structures and simple flexible beams [10-13], PZT materials are introduced into the vibration control of manipulators or mechanisms that only have a single link with a single actuator and sensor bonded $[14,15]$. Meanwhile, compared with numerical simulation, the experimental study of the active vibration control of flexible manipulators is a more challenging work, especially for flexible parallel robots. Zhang et al. [16] have performed an experimental study of active vibration control of 3PRR flexible parallel robots, for which the elastic vibration of flexible links during motion is suppressed by an SRF 
controller. The KED assumption is utilized. Zhang et al. [17] address the dynamic modeling and efficient modal control of a planar parallel manipulator (PPM) with three flexible linkages actuated by linear ultrasonic motors (LUSM). We performed studied dynamic modeling and dynamic analysis of planar 3-RRR flexible parallel robots [18-21].

The residual vibrations of the flexible links of planar 3RRR flexible parallel robots are studied in this paper. In fact, the amplitudes of the residual vibration are greater than the elastic vibration during the motion and will be verified in the experimental study of this paper, and these residual vibrations influence the repeat positioning accuracy of the system seriously. Thus, it is important to suppress the residual vibration of flexible links to improve the repeat positioning accuracy of the system. Essentially, the dynamic responses measured by PZT sensors include unmodeled or unknown dynamics. Therefore, the vibrations of the flexible elements are very complicated and contain many frequency components that are closely spaced. To measure the modal coordinates or velocities more precisely, it is desirable to use as many PZT sensors as possible. However, the number of PZT sensors is limited by the available space of the flexible links and the real-time computing power of the computer. A trade-off must be made between the real-time computing power of the computer hardware and the number of sensors [22].

To solve the above problems, an experimental study of the active vibration control of planar 3-RRR parallel robots with three flexible links, each of which bonds with two pairs of PZT actuators and one PZT sensor film, is implemented in this paper. The moving platform of the planar parallel robots moves along a given trajectory, control experiments are implemented in real-time, and the controller is activated when rigid motion stops. The active control system consists of an industrial computer, a dSPACE controller with many I/O ports, smart flexible links with PZT actuators and PZT sensors, and a PZT driven power and charge amplifier. A strain rate feedback control algorithm is adopted to suppress the elastic vibration of the flexible links of the system [20].

The remainder of the paper is organized as follows. The rigid-elastic coupling dynamic model is established in Section 2. According to the direct and inverse piezoelectric equation of the PZT material, a strain rate feedback control algorithm is given. Section 3 introduces an experimental system setup and describes the working principle of the controller system and each component. In Section 4, the results of an experiment of vibration control are presented. Four experiment schemes are designed. The control performance of the four experiment schemes is analyzed; the experimental results verify the validity of the strain rate feedback controller for suppressing the residual vibration of flexible links. Finally, the conclusions are given in Section 5.

\section{Dynamic Model of Planar 3-RRR Flexible Parallel Robots}

2.1. Sketch of Planar 3-RRR Parallel Robots. A sketch of the planar flexible 3-RRR parallel robots is constructed by the regular triangle moving platform $C_{1} C_{2} C_{3}$, the static platform, and three symmetrical kinematic chains, $A_{1} B_{1} C_{1}, A_{2} B_{2} C_{2}$, and $A_{3} B_{3} C_{3}$, as shown in Figure 2. Each kinematic chain has one active revolute $(\underline{R})$ joint, followed by two consecutive passive revolute $(\mathrm{R})$ joints. The active revolute joints are installed at $A_{i}, i=1,2,3 . A_{1}, A_{2}$, and $A_{3}$ are the regular triangle's three vertices. $A_{1} B_{1}=A_{2} B_{2}=A_{3} B_{3}$ and $B_{1} C_{1}=$ $B_{2} C_{2}=B_{3} C_{3}$. The vertices $O$ and $P$ are centers of the regular triangles $A_{1} A_{2} A_{3}$ and $C_{1} C_{2} C_{3}$, respectively. $O-X Y$ is the global fixed frame. The parameters $\alpha_{i}$ and $\beta_{i}(i=1,2,3)$ are the angles between the $x$-axis of the fixed frame and linkages $A_{i} B_{i}$ and $B_{i} C_{i}(i=1,2,3)$, respectively. $\theta$ is the angle between the $x$-axis of the fixed frame and side $C_{1} C_{2}$ of the regular triangle $C_{1} C_{2} C_{3} . L_{1}, L_{2}, L_{3}$, and $L_{4}$ are the lengths of the segments $A_{i} B_{i}, B_{i} C_{i}, C_{i} P$, and $O A_{i}(i=1,2,3)$.

2.2. Motion Equations of the Beam Element. The flexible link can be modeled by connecting a series of beam elements. Figure 2 shows a beam element before and after deformation. $O-X Y$ is the global fixed frame, and the $A-x y$ is the local moving frame, with the $A x$-axis coincident with the neutral line of the beam element. The original point $A$ is located at one node of the beam element before deformation. $B$ is another node of the beam element. The $O-x^{\prime} y^{\prime}$ system is an intermediate coordinate frame, the origin of which is rigidly attached to the origin of the $O-X Y$ and the axes of which are parallel to the axes of the local moving frame $A-x y . \varphi$ is the angle between the global fixed frame $O-X Y$ and the intermediate coordinate frame $O-x^{\prime} y^{\prime}$.

Considering the general point $C(x, y)$ in the element, let point $C_{0}$ be the corresponding point on the neutral line. Points $C^{\prime}$ and $C_{0}^{\prime}$ are their respective positions after deformation. The elastic deformation of the point $C_{0}$ in $A-x y$ is given by

$$
\mathbf{v}_{0}(x, t)=\left[\begin{array}{c}
\mathbf{v}_{10} \\
\mathbf{v}_{20}
\end{array}\right]=\overline{\mathbf{N}} \mathbf{e}_{f},
$$

where $\mathbf{e}_{f}=\left[\begin{array}{llllllll}e_{1} & e_{2} & e_{3} & e_{4} & e_{5} & e_{6} & e_{7} & e_{8}\end{array}\right]^{\mathrm{T}}$ is the nodal displacement vector, in which $e_{1}$ and $e_{5}$ are the axial displacements of two nodes $A$ and $B$, respectively; $e_{2}$ and $e_{6}$ are the lateral displacements; $e_{3}$ and $e_{7}$ are the elastic rotational angles; $e_{4}$ and $e_{8}$ are the section curvatures; and $\overline{\mathbf{N}}$ is the shape function matrix. Assuming that the axial displacement $\mathbf{v}_{10}$ of point $C_{0}$ is a linear function, the lateral displacement $\mathbf{v}_{20}$ is a fifth-order hermit function, yielding

$$
\overline{\mathbf{N}}=\left[\begin{array}{l}
\overline{\mathbf{N}}_{1} \\
\overline{\mathbf{N}}_{2}
\end{array}\right]=\left[\begin{array}{cccccccc}
f_{1} & 0 & 0 & 0 & f_{5} & 0 & 0 & 0 \\
0 & f_{2} & f_{3} & f_{4} & 0 & f_{6} & f_{7} & f_{8}
\end{array}\right] \text {, }
$$

where

$$
\begin{aligned}
& f_{1}=1-\xi \\
& f_{2}=1-10 \xi^{3}+15 \xi^{4}-6 \xi^{5}, \\
& f_{3}=l\left(\xi-6 \xi^{3}+8 \xi^{4}-3 \xi^{5}\right), \\
& f_{4}=\frac{l^{2}\left(\xi^{2}-3 \xi^{3}+3 \xi^{4}-\xi^{5}\right)}{2},
\end{aligned}
$$




$$
\begin{aligned}
& f_{5}=\xi \\
& f_{6}=10 \xi^{3}-15 \xi^{4}+6 \xi^{5}, \\
& f_{7}=l\left(-4 \xi^{3}+7 \xi^{4}-3 \xi^{5}\right), \\
& f_{8}=\frac{l^{2}\left(\xi^{3}-2 \xi^{4}+\xi^{5}\right)}{2}, \quad \xi=\frac{x}{l} .
\end{aligned}
$$

The deformation displacements of $C$ in $A-x y$ can be written as [23]

$$
\mathbf{v}(x, t)=\left[\begin{array}{l}
v_{1}(x, t) \\
v_{2}(x, t)
\end{array}\right]=\left[\begin{array}{c}
v_{10}-y \frac{\partial v_{20}}{\partial x} \\
v_{20}
\end{array}\right] .
$$

Let $\mathbf{N}=\left[\begin{array}{c}\overline{\mathbf{N}}_{1}-y\left(\partial \overline{\mathbf{N}}_{2} / \partial x\right) \\ \overline{\mathbf{N}}_{2}\end{array}\right]$. Then, the displacement of $C^{\prime}$ can be expressed in the global fixed frame by

$$
\mathbf{r}_{C^{\prime}}=\mathbf{r}_{A}+\mathbf{R}\left(\mathbf{r}_{0}+\mathrm{Ne}_{f}\right)
$$

where $\mathbf{R}$ is the direction cosine matrix, that is, the $O$ $X Y$ system with respect to the $A-x y$ system, and is given by $\mathbf{R}=\left[\begin{array}{cc}\cos \varphi & -\sin \varphi \\ \sin \varphi & \cos \varphi\end{array}\right]$, where the vector $\mathbf{r}_{0}$ is the location coordinates of the point $C$ in the $A-x y$ system and is given by $\left[\begin{array}{ll}x & y\end{array}\right]^{\mathrm{T}}$, where the superscript $\mathrm{T}$ indicates the matrix transpose and the coupling quantity $-y\left(\partial \mathbf{N}_{2}^{\mathrm{T}} / \partial x\right)$ is the axial displacement caused by the transverse displacement of beam. The parameter $\dot{\mathbf{r}}_{C^{\prime}}$ can be obtained by taking the first derivative on

$$
\dot{\mathbf{r}}_{C^{\prime}}=\dot{\mathbf{r}}_{A}+\mathbf{R}_{\varphi}\left(\mathbf{r}_{0}+\mathbf{N e}_{f}\right) \dot{\varphi}+\mathbf{R N} \dot{e}_{f},
$$

where $\mathbf{R}_{\varphi}=\partial \mathbf{R} / \partial \varphi$.

Equation (6) can be expressed compactly as

$$
\dot{\mathbf{r}}_{C^{\prime}}=\left[\begin{array}{lll}
\mathbf{I} & \mathbf{R}_{\varphi}\left(\mathbf{r}_{0}+\mathbf{N e}\right. & \mathbf{R N}
\end{array}\right]\left[\begin{array}{c}
\dot{\mathbf{r}}_{A} \\
\dot{\varphi} \\
\dot{\mathbf{e}}_{f}
\end{array}\right]=\mathbf{S} \dot{\mathbf{q}}_{\mathbf{e}},
$$

where $\mathbf{q}_{e}=\left[\begin{array}{lll}\mathbf{r}_{A}^{\mathrm{T}} & \varphi & \mathbf{e}_{f}^{\mathrm{T}}\end{array}\right]^{\mathrm{T}}$.

2.2.1. Kinetic Energy of the Beam Element. The kinetic energy of the beam element is mainly composed of translational energy and rotational energy. According to (7), the kinetic energy of the beam element is written as

$$
\begin{aligned}
T= & \frac{1}{2} \int_{V} \rho \dot{\mathbf{r}}_{C^{\prime}}^{\mathrm{T}} \dot{\mathbf{r}}_{C^{\prime}} \mathrm{d} V+\frac{1}{2} \int_{0}^{l} \dot{\theta}^{2}(x, t) \mathrm{d} J_{c} \\
& +\frac{1}{2}\left(m_{A} \dot{\mathbf{r}}_{A^{\prime}}^{\mathrm{T}} \dot{\mathbf{r}}_{A^{\prime}}+m_{B} \dot{\mathbf{r}}_{B^{\prime}}^{\mathrm{T}} \dot{\mathbf{r}}_{B^{\prime}}\right) \\
& +\frac{1}{2}\left(J_{A} \dot{\theta}^{2}(0, t)+J_{B} \dot{\theta}^{2}(l, t)\right)=\frac{1}{2} \dot{\mathbf{q}}_{e}^{\mathrm{T}} \mathbf{m}_{e} \dot{\mathbf{q}}_{e},
\end{aligned}
$$

where $\rho$ is the material density, $V$ is the volume of the beam element, $\theta(x, t)$ is the absolute rotation angle of small block $\mathrm{d} x$ in the global fixed frame $O-X Y, \mathrm{~d} J_{c}$ is the moment of inertia about the center of mass of the beam element, $m_{A}$ and $m_{B}$ are the lumped masses of at the ends of the beam element, $J_{A}$ and $J_{B}$ are the lumped moment of inertia at the ends of beam element, and $\theta(0, t)$ and $\theta(l, t)$ are the absolute rotation angle of the two ends of the beam element in the global fixed frame $O-X Y$.

$$
\begin{aligned}
\mathbf{m}_{e}= & {\left[\begin{array}{ccc}
\mathbf{m}_{e r r} & \mathbf{m}_{e r \varphi} & \mathbf{m}_{e r f} \\
& m_{e \varphi \varphi} & \mathbf{m}_{e \varphi f} \\
\text { symmetry } & \mathbf{m}_{e f f}
\end{array}\right], } \\
\mathbf{m}_{e r r}= & \left(m_{d}+m_{A}+m_{B}\right) \mathbf{I}, \\
\mathbf{m}_{e r \varphi}= & \mathbf{R}_{\varphi} \mathbf{N}_{1}+\mathbf{R}_{\varphi} \mathbf{N}_{2} \mathbf{e}_{f}+\mathbf{R}_{\varphi} \overline{\mathbf{N}}_{c} \mathbf{e}, \\
\mathbf{m}_{e r f}= & \mathbf{R N}_{2}+\mathbf{R} \overline{\mathbf{N}}_{c}, \\
\mathbf{m}_{\varphi \varphi}= & \mathbf{N}_{3}+2 \mathbf{N}_{4} \mathbf{e}_{f}+\mathbf{e}_{f}^{\mathrm{T}} \mathbf{N}_{5} \mathbf{e}_{f}+\rho I l+\mathbf{e}^{\mathrm{T}} \widetilde{\mathbf{N}}_{c} \mathbf{e}+J_{A} \\
& +J_{B}, \\
\mathbf{m}_{e \varphi \varphi}= & \mathbf{N}_{3}+2 \mathbf{N}_{4} \mathbf{e}_{f}+\mathbf{e}_{f}^{\mathrm{T}} \mathbf{N}_{5} \mathbf{e}_{f}+\rho I_{z} l+\mathbf{e}^{\mathrm{T}} \widetilde{\mathbf{N}}_{c} \mathbf{e}+J_{A} \\
& +J_{B}, \\
\mathbf{m}_{e \varphi f}= & \mathbf{N}_{6}+\mathbf{e}_{f}^{\mathrm{T}} \mathbf{N}_{7}+\mathbf{e}^{\mathrm{T}} \widetilde{\mathbf{N}_{c}}+\mathbf{C}_{1}, \\
\mathbf{m}_{e f f}= & \mathbf{N}_{5}+\mathbf{C}_{2}+\widetilde{\mathbf{N}}_{c},
\end{aligned}
$$

$\mathbf{N}_{1}=\int_{V} \rho \mathbf{r}_{0} \mathrm{~d} V, \mathbf{N}_{2}=\int_{V} \rho \mathbf{N} \mathrm{d} V, \mathbf{N}_{3}=\int_{V} \rho \mathbf{r}_{0}^{\mathrm{T}} \mathbf{r}_{0} \mathrm{~d} V, \mathbf{N}_{4}=$ $\int_{V} \rho \mathbf{r}_{0}^{\mathrm{T}} \mathbf{N} \mathrm{d} V, \mathbf{N}_{5}=\int_{V} \rho \mathbf{N}^{\mathrm{T}} \mathbf{N} \mathrm{d} V, \mathbf{N}_{6}=\int_{V} \rho \mathbf{r}_{0}^{\mathrm{T}} \widetilde{\mathbf{I}} \mathbf{N} \mathrm{d} V, \mathbf{N}_{7}=$ $\int_{V} \rho \mathbf{N}^{\mathrm{T}} \widetilde{\mathbf{I}} \mathbf{N} \mathrm{d} V, \overline{\mathbf{N}}_{c}=m_{A} \mathbf{N}(0)+m_{B} \mathbf{N}(l), \widetilde{\mathbf{N}}_{c}=m_{A} \mathbf{N}(0)^{\mathrm{T}} \mathbf{N}(0)+$ $m_{B} \mathbf{N}(l)^{\mathrm{T}} \mathbf{N}(l), \widetilde{\overline{\mathbf{N}}}_{c}=m_{A} \mathbf{N}(0)^{\mathrm{T}} \widetilde{\mathbf{I}} \mathbf{N}(0)+m_{B} \mathbf{N}(l)^{\mathrm{T}} \widetilde{\mathbf{I}} \mathbf{N}(l), \mathbf{C}_{1}=$ $\int_{0}^{l} \rho I \mathbf{N}_{2}^{\prime \mathrm{T}} \mathrm{d} x+J_{A} \mathbf{N}_{2}^{\prime \mathrm{T}}(0)+J_{B} \mathbf{N}_{2}^{\prime \mathrm{T}}(l), \mathbf{C}_{2}=\int_{0}^{l} \rho I \mathbf{N}_{2}^{\prime} \mathbf{N}_{2}^{\prime \mathrm{T}} \mathrm{d} x+$ $J_{A} \mathbf{N}_{2}^{\prime}(0) \mathbf{N}_{2}^{\prime \mathrm{T}}(0)+J_{B} \mathbf{N}_{2}^{\prime}(l) \mathbf{N}_{2}^{\prime \mathrm{T}}(l), \widetilde{\mathbf{I}}$ is a skew symmetric matrix defined as

$$
\widetilde{\mathbf{I}}=\left[\begin{array}{cc}
0 & -1 \\
1 & 0
\end{array}\right]
$$

$I_{c}$ is the section moment of inertia of the beam element, and $I$ is the two-order unit matrix.

2.2.2. Strain Energy of the Beam Element. Nonlinear terms in the strain-displacement relationship are neglected [24]. Thus, the strain energy of the beam element can be written as

$$
\begin{aligned}
U_{e} & =\frac{1}{2} \int_{0}^{l} E I(x)\left(v_{20}^{\prime \prime}\right)^{2} \mathrm{~d} x+\frac{1}{2} \int_{0}^{l} E S(x)\left(v_{10}^{\prime}\right)^{2} \mathrm{~d} x \\
& =\frac{1}{2} \mathbf{q}_{e}^{\mathrm{T}} \mathbf{k}_{e} \mathbf{q}_{e},
\end{aligned}
$$

where $v_{10}^{\prime}=\partial v_{10} / \partial x, v_{20}^{\prime \prime}=\partial^{2} v_{20} / \partial x^{2}$, and $E$ is the elastic modulus of materials. $I(x)$ and $S(x)$ are the cross-sectional 
moment of inertia and the cross-sectional area of the beam element, respectively.

$$
\begin{aligned}
\mathbf{k}_{e}= & {\left[\begin{array}{ccc}
\mathbf{0} & \mathbf{0} & \mathbf{0} \\
\mathbf{0} & 0 & \mathbf{0} \\
\mathbf{0} & \mathbf{0} & \mathbf{k}_{e f f}
\end{array}\right], } \\
\mathbf{k}_{e f f}= & \int_{0}^{l} E I(x) \mathbf{N}_{2}^{\prime \prime}(x) \mathbf{N}_{2}^{\prime \prime}(x) \mathrm{d} x \\
& +\int_{0}^{l} E S(x) \mathbf{N}_{1}^{\prime \mathrm{T}}(x) \mathbf{N}_{1}^{\prime}(x) \mathrm{d} x .
\end{aligned}
$$

2.2.3. Motion Equations of the Beam Element. According to Lagrange's equation, the dynamic equation of the beam element can be derived as

$$
\mathbf{m}_{e} \ddot{\mathbf{q}}_{e}+\mathbf{k}_{e} \mathbf{q}_{e}=\mathbf{p}_{e, e}+\mathbf{p}_{e, v},
$$

$$
\begin{aligned}
& \mathbf{p}_{e, v}=-\dot{\mathbf{m}} \dot{\mathbf{q}}+\frac{\partial}{\partial \mathbf{q}}\left(\frac{1}{2} \dot{\mathbf{q}}^{\mathrm{T}} \mathbf{m} \dot{\mathbf{q}}\right)=\left[\begin{array}{c}
-\dot{\varphi}^{2} \mathbf{R}_{\varphi \varphi} \mathbf{N}_{1}-\dot{\varphi}^{2} \mathbf{R}_{\varphi \varphi} \mathbf{N}_{2} \mathbf{e}_{f}-2 \dot{\varphi} \mathbf{R}_{\varphi} \mathbf{N}_{2} \dot{\mathbf{e}}_{f}-\dot{\varphi}^{2} \mathbf{R}_{\varphi \varphi} \overline{\mathbf{N}}_{c} \mathbf{e}-2 \dot{\varphi} \mathbf{R}_{\varphi} \overline{\mathbf{N}}_{c} \dot{\mathbf{e}}_{f} \\
-2\left(\mathbf{N}_{4} \dot{\mathbf{e}}_{f}+\mathbf{e}_{f}^{\mathrm{T}} \mathbf{N}_{5} \dot{\mathbf{e}}_{f}+\mathbf{e}^{\mathrm{T}} \widetilde{\mathbf{N}} \dot{\mathbf{e}}_{f}\right) \dot{\varphi}-\left(\dot{\mathbf{e}}_{f}^{\mathrm{T}} \mathbf{N}_{7}+\mathbf{e}_{f}^{\mathrm{T}} \widetilde{\mathbf{N}}_{c}\right) \dot{\mathbf{e}}_{f} \\
2 \mathbf{N}_{7} \dot{\mathbf{e}}_{f} \dot{\varphi}+\left(\mathbf{N}_{4}^{\mathrm{T}}+\mathbf{N}_{5} \mathbf{e}_{f}\right) \dot{\varphi}^{2}+2 \widetilde{\mathbf{N}}_{c} \dot{e}_{f} \dot{\varphi}+\widetilde{\mathbf{N}}_{c} \dot{e}^{2}
\end{array}\right], \\
& \mathbf{p}_{e, e}=\left[\begin{array}{lll}
\mathbf{F}^{\mathrm{T}} & \mathbf{F}^{\mathrm{T}} \mathbf{R}_{\varphi}\left(\mathbf{r}_{0}+\mathbf{N} \mathbf{e}_{f}\right) & \mathbf{F}^{\mathrm{T}} \mathbf{R} \mathbf{N}
\end{array}\right]^{\mathrm{T}} .
\end{aligned}
$$

where $\mathbf{p}_{e, e}$ and $\mathbf{p}_{e, v}$ are the generalized external forces and the quadratic velocity vector that contains the gyroscopic and the Coriolis force components, respectively.
The elemental dynamic equation has been established in the $A$-xy system. Before forming the dynamic equation of the system, (13) must be expressed in the $O-X Y$ system. Defining the coordinate transformation matrix $\mathbf{B}$

$$
\mathbf{B}=\left[\begin{array}{cccccccc}
\cos \varphi & \sin \varphi & 0 & 0 & 0 & 0 & 0 & 0 \\
-\sin \varphi & \cos \varphi & 0 & 0 & 0 & 0 & 0 & 0 \\
0 & 0 & 1 & 0 & 0 & 0 & 0 & 0 \\
0 & 0 & 0 & 1 & 0 & 0 & 0 & 0 \\
0 & 0 & 0 & 0 & \cos \varphi & \sin \varphi & 0 & 0 \\
0 & 0 & 0 & 0 & -\sin \varphi & \cos \varphi & 0 & 0 \\
0 & 0 & 0 & 0 & 0 & 0 & 1 & 0 \\
0 & 0 & 0 & 0 & 0 & 0 & 0 & 1
\end{array}\right] .
$$

Let

$$
\mathbf{e}_{f}=\mathbf{B E}_{f},
$$

where $\mathbf{E}_{f}$ is the elemental nodal coordinate vector in the $O$ $X Y$ system. By taking the first and the second derivatives of (16) with respect to time,

$$
\begin{aligned}
& \dot{\mathbf{e}}_{f}=\mathbf{B}_{\varphi} \mathbf{E}_{f} \dot{\varphi}+B \dot{\mathbf{E}}_{f}, \\
& \ddot{\mathbf{e}}_{f}=\mathbf{B}_{\varphi \varphi} \mathbf{E}_{f} \dot{\varphi}^{2}+2 \mathbf{B}_{\varphi} \dot{\mathbf{E}}_{f} \dot{\varphi}+\mathbf{B}_{\varphi} \mathbf{E}_{f} \ddot{\varphi}+\mathbf{B} \ddot{\mathbf{E}}_{f},
\end{aligned}
$$

where $\mathbf{B}_{\varphi}=\partial \mathbf{B} / \partial \varphi$ and $\mathbf{B}_{\varphi \varphi}=\partial^{2} \mathbf{B} / \partial \varphi^{2}$. Substituting (16) and (17) into (13) and premultiplying by matrix $\left[\begin{array}{lll}\mathbf{I} & 0 & \mathbf{0} \\ 0 & 1 & \mathbf{E}_{f}^{\mathrm{T}} \mathbf{B}_{\varphi} \\ 0 & 0 & \mathbf{B}^{\mathrm{T}}\end{array}\right]$, the dynamic equation of the beam element can be expressed in the $O-X Y$ system by

$$
\mathbf{M}_{e} \ddot{\mathbf{U}}_{e}+\mathbf{K}_{e} \mathbf{U}_{e}=\mathbf{Q}_{e, e}+\mathbf{Q}_{e, v},
$$

where $\mathbf{U}_{e}=\left[\begin{array}{lll}\mathbf{r}_{e}^{\mathrm{T}} & \varphi & \mathbf{E}_{f}^{\mathrm{T}}\end{array}\right]^{\mathrm{T}}$ and $\mathbf{M}_{e}$ and $\mathbf{K}_{e}$ are the $8 \times 8$ element generalized mass and the stiffness matrices in the $O$ $X Y$ system, respectively. $\mathbf{Q}_{e, v}$ is the $8 \times 1$ quadratic velocity vector in the $O-X Y$ system. $\mathbf{Q}_{e, e}$ is the $8 \times 1$ generalized external forces vector in the $O-X Y$ system.

2.3. Constraint Equations. Different from the simple structure, the constraint relationships of the planar 3-RRR of flexible parallel robots, which include the rigid-body motion constraints, elastic deformation motion constraints, and dynamic constraints of the moving platform, are very complex and can be used to eliminate the correlation of the generalized coordinates.

2.3.1. Constraint Equations of the Rigid-Body Motion. The generalized coordinates are formed by rigid-body motion coordinates and elastic coordinates. As shown in Figure 1, the rigid-body motion coordinates include drive joint rotation angles $\boldsymbol{\alpha}=\left[\begin{array}{lll}\alpha_{1} & \alpha_{2} & \alpha_{3}\end{array}\right]^{\mathrm{T}}$, passive joint rotation angles $\boldsymbol{\beta}=\left[\begin{array}{lll}\beta_{1} & \beta_{2} & \beta_{3}\end{array}\right]^{\mathrm{T}}$, and translation displacement and rotation angle of the moving platform $\mathbf{X}_{P}=\left[\begin{array}{lll}X_{P} & Y_{P} & \theta\end{array}\right]^{\mathrm{T}}$, and rigid-body motion coordinate vectors $\boldsymbol{\alpha}, \boldsymbol{\beta}$, and $\mathbf{X}_{P}$ are not independent. Because three kinematic chains of 3-RRR parallel robots are full symmetrical chains, only one chain is studied. As shown in Figure 3, a closed-loop vector equation can be established:

$$
\overrightarrow{O A_{i}}+\overrightarrow{A_{i} B_{i}}+\overrightarrow{B_{i} C_{i}}+\overrightarrow{C_{i} P}-\overrightarrow{O P}=\overrightarrow{0}
$$

Projecting (19) into $X$ and $Y$ components yields

$$
\begin{aligned}
& X_{A_{i}}+L_{1} \cos \alpha_{i}+L_{2} \cos \beta_{i} \\
& \quad+L_{3} \cos \left(\frac{\pi}{6}+\frac{2(i-1) \pi}{3}+\theta\right)-X_{P}=0,
\end{aligned}
$$




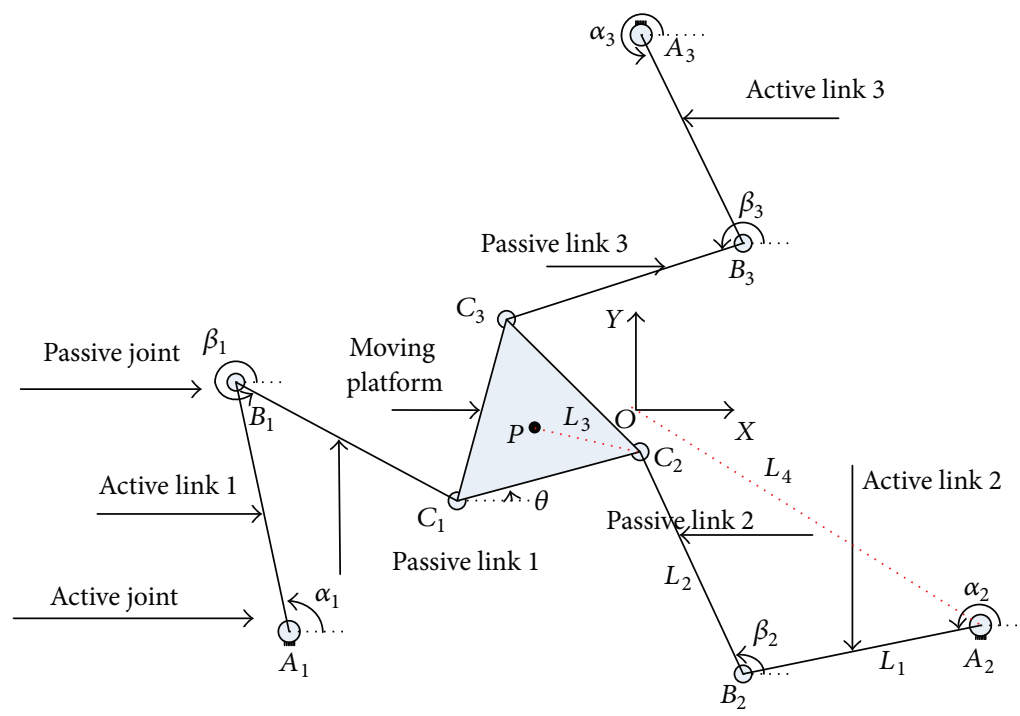

FIGURE 1: The sketch of planar 3-RRR parallel robot.

$Y_{A_{i}}+L_{1} \sin \alpha_{i}+L_{2} \sin \beta_{i}$

$$
+L_{3} \sin \left(\frac{\pi}{6}+\frac{2(i-1) \pi}{3}+\theta\right)-Y_{P}=0,
$$

$$
i=1,2,3,
$$

where $\left(X_{A_{i}}, Y_{A_{i}}\right)$ and $\left(X_{P}, Y_{P}\right)$ are the coordinates of the points $A_{i}$ and $P$ in the $O-X Y$ system, respectively.

\subsubsection{Constraint Equations of the Elastic Deformation Motion.} As shown in Figure 3, center point $P$ and vertex $C_{i}$ of the moving platform are moved to points $P^{\prime}$ and $C_{i}^{\prime}$ because of the elastic deformation motion of the flexible links $A_{i} B_{i}$ and $B_{i} C_{i}(i=1,2,3) . P-x y$ is the local coordinate system with original point $P$, and $P^{\prime}-x^{\prime} y^{\prime}$ is the elastic coordinate system with original point $P^{\prime}$.T, $\widetilde{\mathbf{T}}$, and $\overline{\mathbf{T}}$ are the coordinate transformation matrices for $p-x y \rightarrow O-X Y, p^{\prime}-x^{\prime} y^{\prime} \rightarrow p$ $x y$, and $p^{\prime}-x^{\prime} y^{\prime} \rightarrow O-X Y$, respectively. Thus, $\overline{\mathbf{T}}=\mathbf{T} \widetilde{\mathbf{T}}$. $U_{i_{22}}, U_{i_{23}}$, and $U_{i_{24}}$ are the elastic displacements and elastic rotational angle at the end point $C_{i}$ of the flexile link $B_{i} C_{i}$; the section curvature is equal to zero at point $C_{i} . \Delta X_{P}$ and $\Delta Y_{P}, \varepsilon$ are the translational and rotational declinations of the moving platform because of the elastic vibration of the flexible links. Assuming that the moving platform is rigid, $P C_{i}=P^{\prime} C_{i}^{\prime}$. Note that

$$
\begin{aligned}
& \mathbf{T}=\left[\begin{array}{ccc}
\cos \theta & -\sin \theta & X_{P} \\
\sin \theta & \cos \theta & Y_{P} \\
0 & 0 & 1
\end{array}\right], \\
& \widetilde{\mathbf{T}}=\left[\begin{array}{ccc}
1 & -\varepsilon & \Delta X_{P} \\
\varepsilon & 1 & \Delta Y_{P} \\
0 & 0 & 1
\end{array}\right] .
\end{aligned}
$$

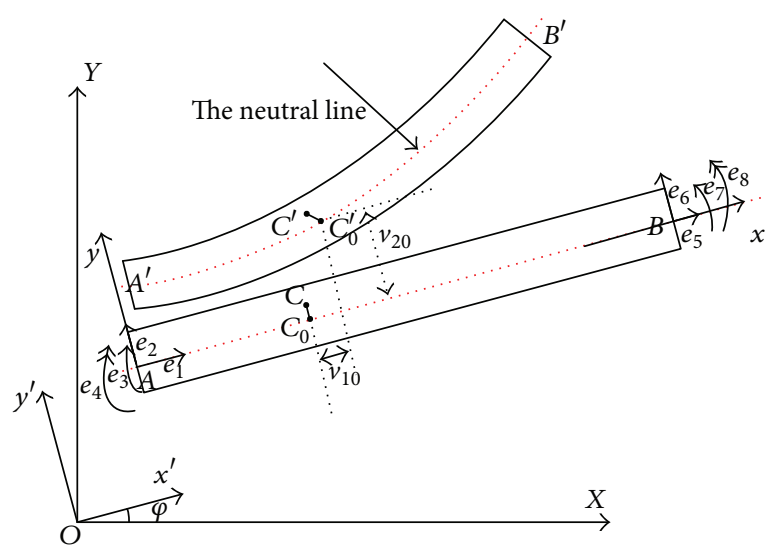

FIGURE 2: Beam element deformation.

Assuming that $\left(\begin{array}{lll}X_{C_{i}} & Y_{C_{i}}\end{array}\right)$ and $\left(\begin{array}{ll}X_{C_{i}^{\prime}} & Y_{C_{i}^{\prime}}\end{array}\right)$ are the coordinates of the points $C_{i}, C_{i}^{\prime}$ in the $O-X Y$ system yields

$$
\begin{aligned}
& {\left[\begin{array}{c}
X_{C_{i}^{\prime}} \\
Y_{C_{i}^{\prime}} \\
1
\end{array}\right]=\overline{\mathbf{T}}\left[\begin{array}{c}
x_{C_{i}^{\prime}}^{\prime} \\
y_{C_{i}^{\prime}}^{\prime} \\
1
\end{array}\right]_{P^{\prime}},} \\
& {\left[\begin{array}{c}
X_{C_{i}} \\
Y_{C_{i}} \\
1
\end{array}\right]=\mathbf{T}\left[\begin{array}{c}
x_{C_{i}} \\
y_{C_{i}} \\
1
\end{array}\right]_{P},}
\end{aligned}
$$

where $\left(x_{C_{i}}, y_{C_{i}}\right)_{p}=\left(x_{C_{i}^{\prime}}^{\prime}, y_{C_{i}^{\prime}}^{\prime}\right)_{p^{\prime}}=\left(L_{3} \cos (\pi / 2+2 i \pi / 3)\right.$, $\left.L_{3} \sin (\pi / 2+2 i \pi / 3)\right)$. ()$_{p}$ denotes the description in $p-x y$. 


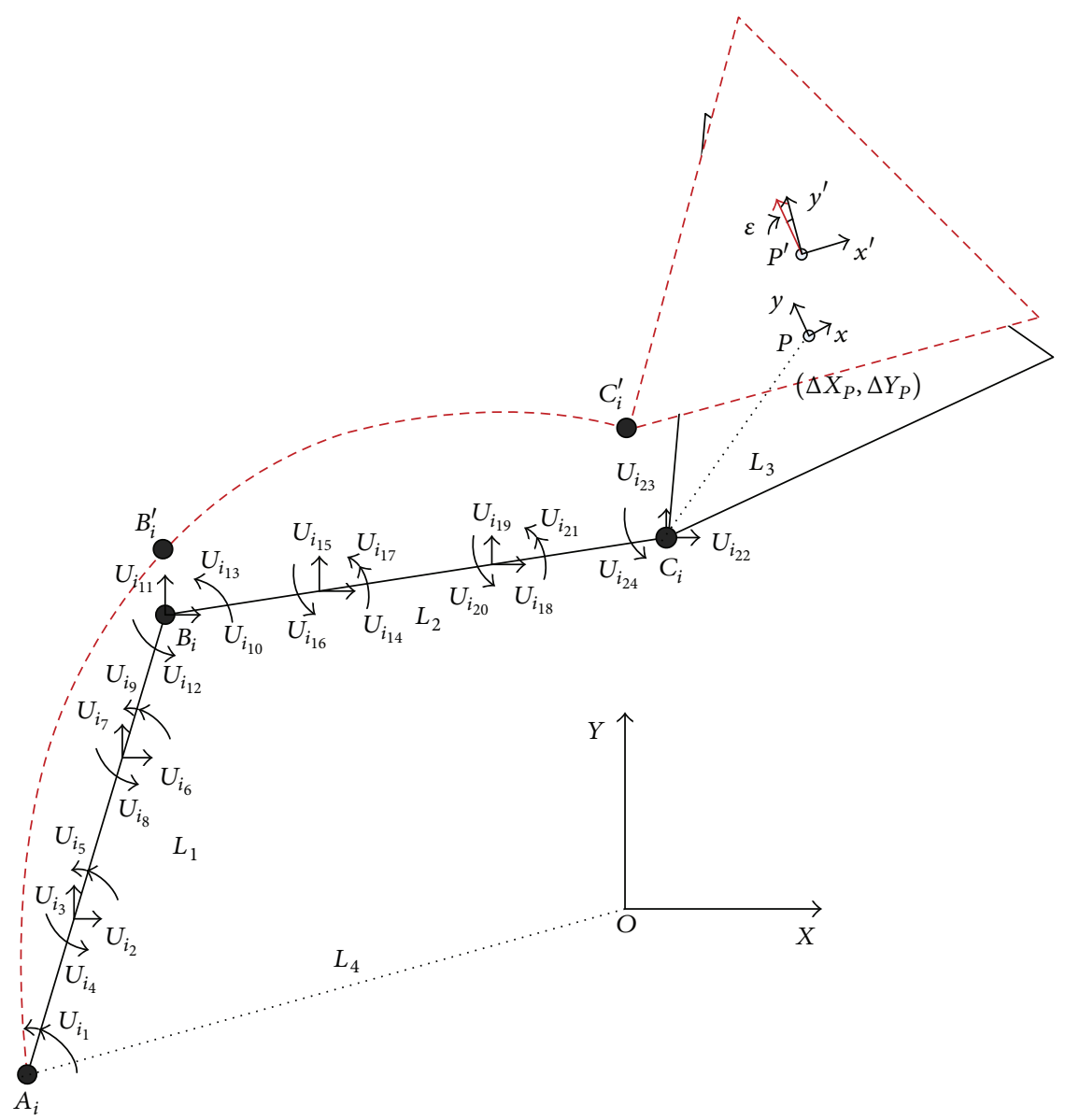

FIGURE 3: The flexible kinematic chain.

Setting $\mathbf{T}_{1}=\left[\begin{array}{cc}\cos \theta & -\sin \theta \\ \sin \theta & \cos \theta\end{array}\right]$ yields

$$
\begin{aligned}
{\left[\begin{array}{c}
U_{i_{22}} \\
U_{i_{23}}
\end{array}\right] } & =\left[\begin{array}{c}
X_{C_{i}^{\prime}} \\
Y_{C_{i}^{\prime}}
\end{array}\right]-\left[\begin{array}{c}
X_{C_{i}} \\
Y_{C_{i}}
\end{array}\right] \\
& =\left[\begin{array}{ll}
\mathbf{I} & \mathbf{T}_{1}\left(\begin{array}{c}
-y_{C_{i}} \\
x_{C_{i}}
\end{array}\right)_{P}
\end{array}\right]\left[\begin{array}{c}
\Delta X_{P} \\
\Delta Y_{P} \\
\varepsilon
\end{array}\right], \\
\varepsilon & =\sum_{i=1}^{3} U_{i_{24}},
\end{aligned}
$$

where $\mathbf{I}$ is the $2 \times 2$ unit matrix.

2.3.3. Dynamic Constraints of the Moving Platform. Assuming that $\mathbf{F}_{i}$ is the generalized joint constraint reaction force that the passive joint $C_{i}$ exerts on the moving platform, $M_{v}$ and $J_{v}$ are the mass and moment of inertia of the moving platform, respectively. Thus, the dynamic constraint of the moving platform can be expressed by

$$
\left[\begin{array}{ccc}
M_{v} & & \\
& M_{v} & \\
& & J_{v}
\end{array}\right]\left[\begin{array}{c}
\Delta \ddot{X}_{P} \\
\Delta \ddot{Y}_{P} \\
\ddot{\varepsilon}
\end{array}\right]=\sum_{i=1}^{3} \mathbf{F}_{i}-\left[\begin{array}{c}
M_{v} \ddot{X}_{P} \\
M_{v} \ddot{Y}_{P} \\
J_{v} \ddot{\varepsilon}
\end{array}\right] .
$$

2.4. Motion Equations of Planar 3-RRR Parallel Robots. Considering the above constraint (20)-(24), let $\mathbf{U}$ be the generalized elastic coordinate vector of all of the flexible links and the moving platform in the $O-X Y$ system; namely, $\mathbf{U}=\left[\begin{array}{lllll}U_{11} & \cdots & U_{3 n} & \Delta X_{P} & \Delta Y_{P}\end{array}\right]^{\mathrm{T}}$. By assembling all of the elements in (18), according to the compatibility at the nodes, the equations of motion of the planar 3-RRR parallel robots are given as

$$
\mathbf{M U ̈}+\mathbf{C U}+\mathbf{K U}=\mathbf{Q}
$$

where $\mathbf{M}, \mathbf{C}$, and $\mathbf{K}$ are the generalized mass, damping, and stiffness matrices, respectively; $\mathbf{Q}$ includes the gyroscopic force, the Coriolis force components, and the generalized external force.

\section{Strain Rate Feedback Control Algorithm}

Due to the direct and inverse piezoelectric effect of PZT material, PZT material is widely used for designing active vibration controllers of flexible multibody systems. Assuming that the PZT sensor film is perfectly bonded onto the flexible 


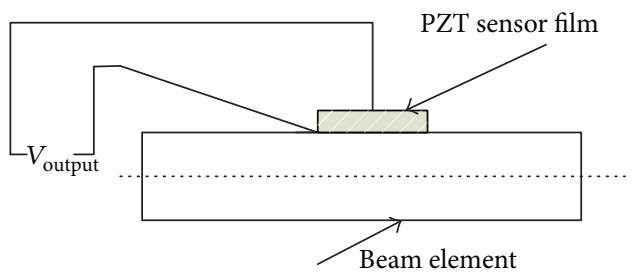

FIGURE 4: One PZT sensor film is bonded on beam element.

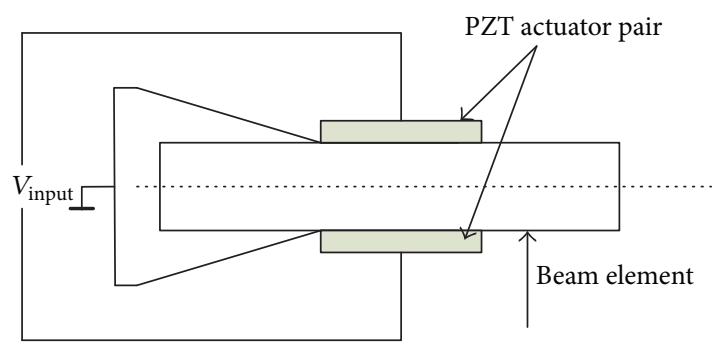

Figure 5: One PZT actuator pair is bonded on beam element.

beam, as shown in Figure 4, the direct piezoelectric equation of PZT sensor can be expressed as [22]

$$
V_{s e}=k_{s e}\left(\frac{\partial v_{2}^{2}\left(x_{k}, t\right)}{\partial x^{2}}\right)=k_{s e} \mathbf{N}_{2}^{\prime \prime T}\left(x_{k}\right) \mathbf{e}_{f},
$$

where $k_{s e}=\left(E_{s} d_{31} w_{s} / C_{s}\right)\left(L_{s}+L_{e}\right), E_{s}$ is Young's modulus of the piezoelectric sensor, $d_{31}$ is the piezoelectric constant, $w_{s}$ is the width of the piezoelectric sensor, $L_{s}$ is the length of the piezoelectric sensor, $C_{s}$ is the capacitance of the piezoelectric sensor, $L_{e}$ is the length of the beam element, $x_{k}$ is the $x$ direct coordinates of the midpoint of the $k$ th sensor in the element coordinate frame, $\mathbf{N}_{2}(x)$ is the column vector of the 2 nd line of $\mathbf{N}(x)$, and "denotes the second derivative to the variable $x$.

As shown in Figure 5, two of the same PZT actuators are perfectly bonded onto the upper and lower surfaces of the beam element in the same position and the polarization directions of the two PZT actuators are also the same. Two adhesive surfaces are electrically grounded, and the other two surfaces are linked into input voltage by wire. According to inverse piezoelectric effect of the PZT material, the torque of the two ends of the PZT actuator pair is given by [25]

$$
\begin{aligned}
M_{a e}\left(x_{k_{1}}\right) & =-M_{a e}\left(x_{k_{2}}\right)=-d_{31} E_{p} w_{p}\left(t_{p}+t_{b}\right) V_{\text {input }} \\
& =-K_{a e} V_{a e}
\end{aligned}
$$

where $K_{a e}=d_{31} E_{p} b_{p}\left(t_{p}+t_{b}\right), V_{a e}=V_{\text {input }}, x_{k_{1}}$ is the $x$ direct coordinate of the left end of the $k$ th PZT actuator, and $x_{k_{2}}$ is the $x$ direct coordinate of the right end of the $k \mathrm{th} \mathrm{PZT}$ actuator. $E_{p}$ is Young's modulus of the PZT actuator, $w_{p}$ is the width of the PZT actuator, $t_{p}$ is the thickness of the PZT actuator, and $t_{b}$ is the thickness of the beam element.

Assuming there are $m$ PZT actuator pairs and $p$ PZT sensor films in this system, integrating all of the inverse piezoelectric equations of the PZT actuator pairs and elastodynamics yields

$$
\mathbf{M U ̈}+\mathbf{C U}+\mathbf{K U}=\mathbf{Q}+\overline{\mathbf{K}} \mathbf{V}_{\text {input }},
$$

where $\overline{\mathbf{K}}$ is the input matrix; $\mathbf{V}_{\text {input }}$ is the $m$ dimension input vector.

Assembling all of the direct piezoelectric equations of the PZT sensor films yields

$$
\mathbf{V}_{s}=\mathbf{C}_{s} \mathbf{U}
$$

where $\mathbf{V}_{s}$ is the $p$ dimension output vector and $\mathbf{C}_{s}$ is the output matrix.

According to (28) and (29), the general motion equation of the system can be expressed as

$$
\begin{aligned}
\mathbf{M U}+\mathbf{C U}+\mathbf{K U} & =\mathbf{Q}+\mathbf{K} \mathbf{V}_{\text {input }}, \\
\mathbf{V}_{s} & =\mathbf{C}_{s} \mathbf{U}
\end{aligned}
$$

In this paper, the goal is to suppress the residual vibration of flexible links of planar 3-RRR flexible parallel robots so that the moving platform can accurately stop at a given position. Thus, the mass matrix $\mathbf{M}$ is a positive definite symmetric constant matrix, the stiffness matrix $\mathbf{K}$ is a symmetric constant matrix, and $\mathbf{Q}$ is a zero vector, assuming that the damping matrix $\mathbf{C}$ barely includes structural damping. Considering that residual vibration of the system is decided by its lowerorder modes, the real modal method is utilized for extracted lower-order modes of the controlled system. Assuming that the first $r$ lower-order modes are suppressed, the general motion equation of the system can be simplified as

$$
\begin{aligned}
\ddot{\boldsymbol{\eta}}_{r} & +\operatorname{diag}\left(2 \xi_{1} w_{1}, 2 \xi_{2} w_{2}, \ldots, 2 \xi_{r} w_{r}\right) \dot{\boldsymbol{\eta}}_{r} \\
& +\operatorname{diag}\left(w_{1}^{2}, w_{2}^{2}, \ldots, w_{r}^{2}\right) \boldsymbol{\eta}_{r}=\mathbf{K}_{r} \mathbf{V}_{\text {input }} \\
\mathbf{V}_{s} & =\mathbf{C}_{r} \boldsymbol{\eta}_{r}
\end{aligned}
$$

where $\boldsymbol{\eta}_{r}$ is the first $r$ lower-order mode coordinates.

The strain rate feedback control is adopted to suppress the residual vibration of the flexible links. Here the derivation of output voltage of the sensor is fed back to the corresponding actuators pairs, yielding

$$
\mathbf{V}_{\text {input }}=-\mathbf{K}_{d} \dot{\mathbf{V}}_{s}=-\mathbf{K}_{d} \mathbf{C}_{r} \dot{\boldsymbol{\eta}}_{r}=-\mathbf{K}_{v} \dot{\boldsymbol{\eta}}_{r}
$$

where $\mathbf{K}_{d}$ is feedback gain matrix.

Assuming $\xi_{i}(i=1,2, \ldots, r)$ is the damping ratio coefficients of the $i$ th mode coordinates, then the motion equation of the controlled system can be expressed as

$$
\begin{aligned}
\ddot{\boldsymbol{\eta}}_{r}+ & \left(\operatorname{diag}\left(2 \xi_{1} w_{1}, 2 \xi_{2} w_{2}, \ldots, 2 \xi_{r} w_{r}\right)+\mathbf{K}_{r} \mathbf{K}_{v}\right) \dot{\boldsymbol{\eta}}_{r} \\
& +\operatorname{diag}\left(w_{1}^{2}, w_{2}^{2}, \ldots, w_{r}^{2}\right) \boldsymbol{\eta}_{r}=\mathbf{0} .
\end{aligned}
$$

Assuming that the damping ratio coefficient $\xi_{i}$ is accelerated to $\bar{\xi}_{i}$, the feedback gain matrix can be expressed as

$$
\begin{gathered}
\mathbf{K}_{d}=\mathbf{K}_{r}^{+} \operatorname{diag}\left(2\left(\bar{\xi}_{1}-\xi_{1}\right) w_{1}, 2\left(\bar{\xi}_{2}-\xi_{2}\right)\right. \\
\left.\cdot w_{2}, \ldots, 2\left(\bar{\xi}_{r}-\xi_{r}\right) w_{r}\right) \mathbf{C}_{r}^{+},
\end{gathered}
$$




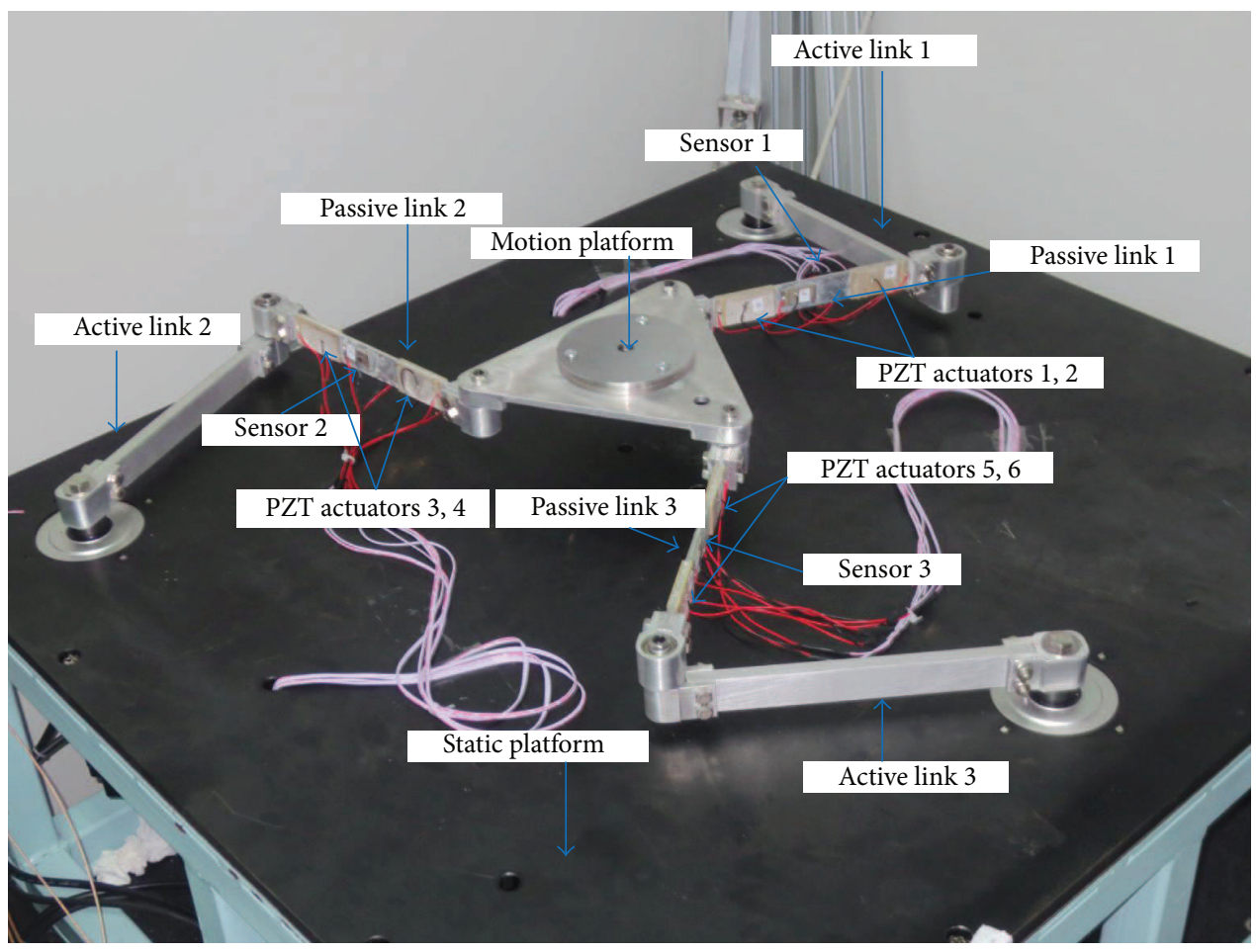

FIgURE 6: Experiment setup of active vibration control system.

where $\mathbf{K}_{r}^{+}$and $\mathbf{C}_{r}^{+}$are the generalized inverse matrices of $\mathbf{K}_{r}$ and $\mathbf{C}_{r}$, respectively.

According to (34), the strain rate feedback control leads to the increase of the damping of the system.

\section{Experimental System Setup}

The experimental setup of the active vibration control system, as shown in Figure 6, consists of a planar 3-RRR flexible parallel mechanism, a static platform, three Yaskawa servomotors, and SHIMPO reducers (reduction ratio is $1: 5$ ). The three passive links are all flexible and identical with dimensions of $252 \mathrm{~mm} \times 25 \mathrm{~mm} \times 3 \mathrm{~mm}$. The three active links are all rigid and identical with dimensions of $254 \mathrm{~mm} \times$ $25 \mathrm{~mm} \times 10 \mathrm{~mm}$. The links are insulated by surface oxidation. The static platform of the planar 3-RRR flexible parallel robots consists of a marble pedestal, a steel-framed structure, and a rectangular steel plate. The experimental setup also includes PZT actuators and PZT sensors, PZT drive power, a charge amplifier, a real-time semiphysical simulation system, dSPACE, and an industrial computer. The dimensions of each PZT actuator are $50 \mathrm{~mm} \times 25 \mathrm{~mm} \times 2 \mathrm{~mm}$, and the dimensions of each PZT sensor are $30 \mathrm{~mm} \times 15 \mathrm{~mm} \times 1 \mathrm{~mm}$. The piezoelectric constant of the piezoelectric actuator/sensor is $d_{31}=1.85 \times 10^{-10} \mathrm{c} / \mathrm{N}$, and Young's modulus of the piezoelectric actuator/sensor is $E_{s}$ and $E_{p}=1.17 \times 10^{6} \mathrm{MP}$. The smart beam shown in Figure 7 has two pairs of PZT actuators symmetrically bonded on the surface of the flexible beam and one PZT sensor is bonded on its middle.

The PZT drive power is manufactured by Harbin Core Tomorrow Science and Technology Co., Ltd., its main module

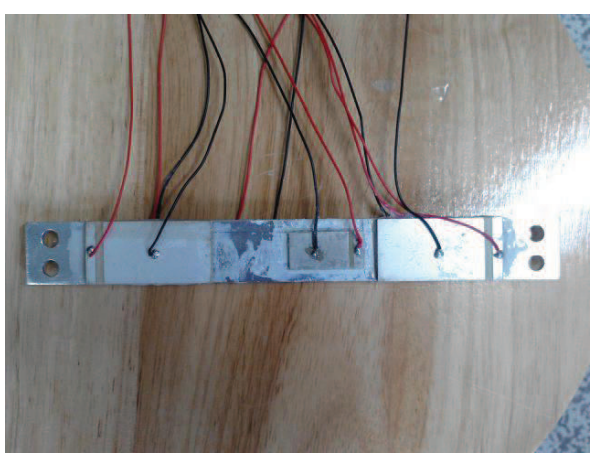

FIGURE 7: A smart flexible beam.

is a 090716 amplifier, its analog input is $\pm 10 \mathrm{~V}$, its analog output voltage is $\pm 150 \mathrm{~V}$, its power is $35 \mathrm{~W}$, its average current is $115 \mathrm{~mA}$, and its working temperature range is $0^{\circ}-50^{\circ}$. The PZT drive power is shown in Figure 8.

Figure 9 shows the YE5850 charge amplifier, which is manufactured by Jiangsu Lianneng Electronic Technology Co., Ltd. The amplifier is a bandwidth charge amplifier whose input voltage is proportional to its output voltage. Because the lower-cut-off frequency of the amplifier is very small, it is suitable for quasistatic calibration of piezoelectric pressure transducer. The maximum input charge and maximum output voltage of the amplifier are $10^{6} \mathrm{PC}$ and $\pm 10 \mathrm{~V}$, respectively.

dSPACE (real-time semiphysical simulation system) is a control system development platform and test platform 


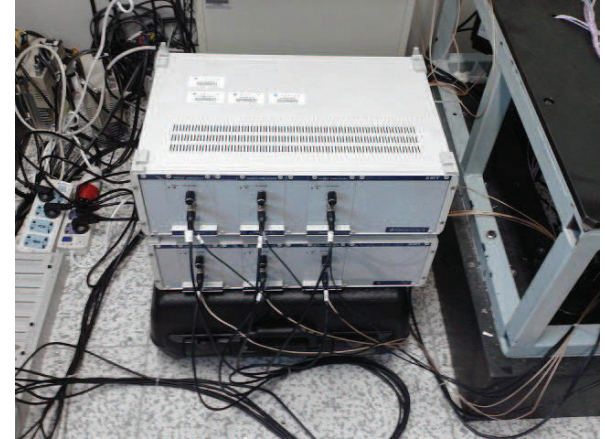

FIgURE 8: PZT drive power.

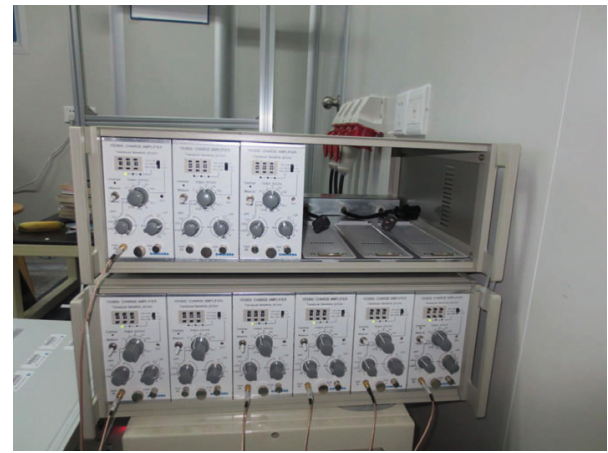

FIgURE 9: Charge amplifier.

based on MATLAB/Simulink. dPACE can achieve a seamless connection with MATLAB/Simulink. dSPACE owns a hardware system that has a high-speed computing capability and friendly software environment for code generating, downloading, and debugging.

The DS1103PPC control card (see Figure 10) and DS2211 HIL I/O panel (see Figure 11) are used in the experiment. The DS1103PPC control card is the most powerful card to date and has abundant $\mathrm{I} / \mathrm{O}$ ports. Figure 12 shows an industrial computer that is manufactured by YANHUA company. DS1103PPC control card is installed in the expansion slot of the industrial computer.

\section{Experimental Results and Analysis}

Assume that the motion trajectory of the moving platform of the system is designed as follows.

First Step. The midpoint of the moving platform moves to the coordinate $(0,0.05)$ from the origin of the fixed coordinate system along the linear trajectory of (35); the process takes 1 second.

Second Step. Move 0.3 seconds along the circular trajectory of (36), and then stop for 2.7 seconds.

Third Step. The second step is repeated 5 times.

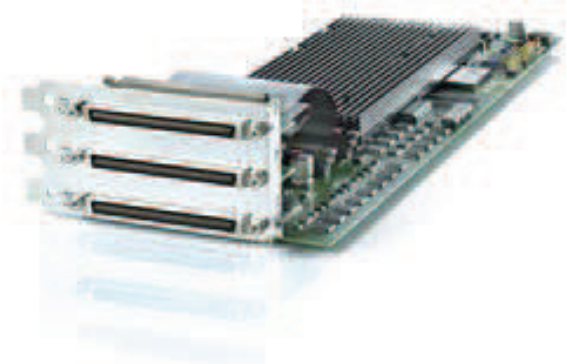

FIGURE 10: dSPACE control card.

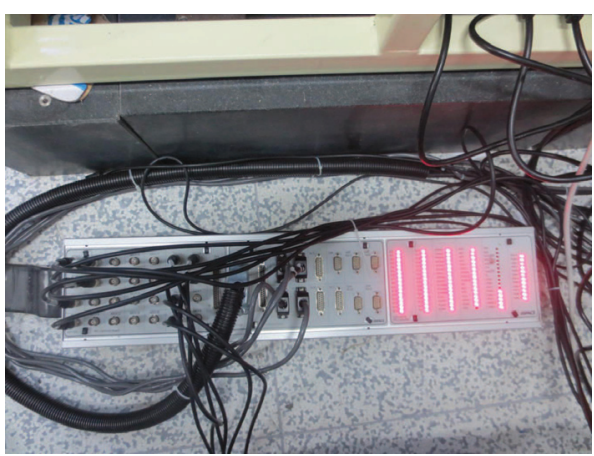

FIGURE 11: dSPACE control panel.

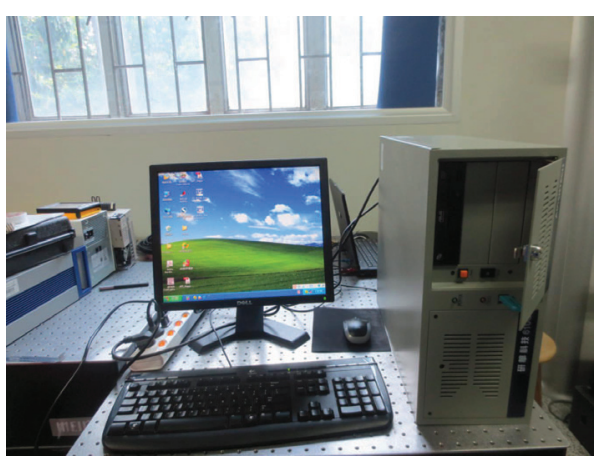

FIGURE 12: Industrial computer.

Fourth Step. The moving platform returns to the initial position along the linear trajectory of (37).

The entire motion period takes 17 seconds.

$$
\begin{aligned}
X_{p} & =0 \\
Y_{p} & =0.05 t-\frac{1}{40 \pi} \sin (2 \pi t), \\
\theta & =0 \\
X_{p} & =0.05 \sin \left(\frac{20 \pi t}{3}\right) \\
Y_{p} & =0.05 \cos \left(\frac{20 \pi t}{3}\right) \\
\theta & =0
\end{aligned}
$$




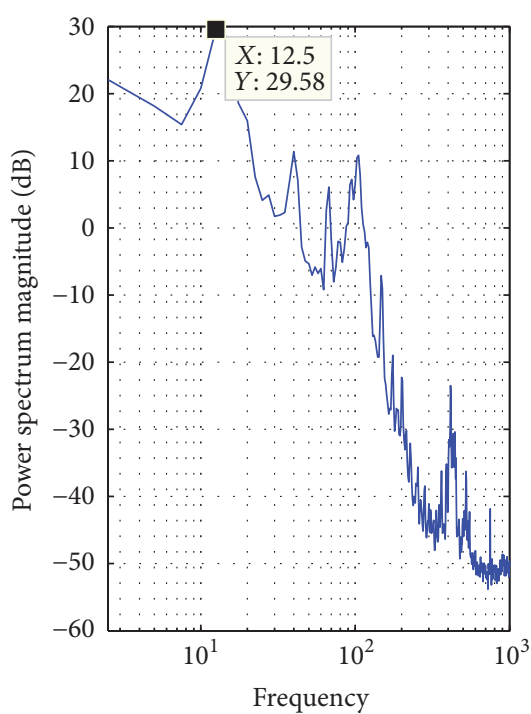

(a)

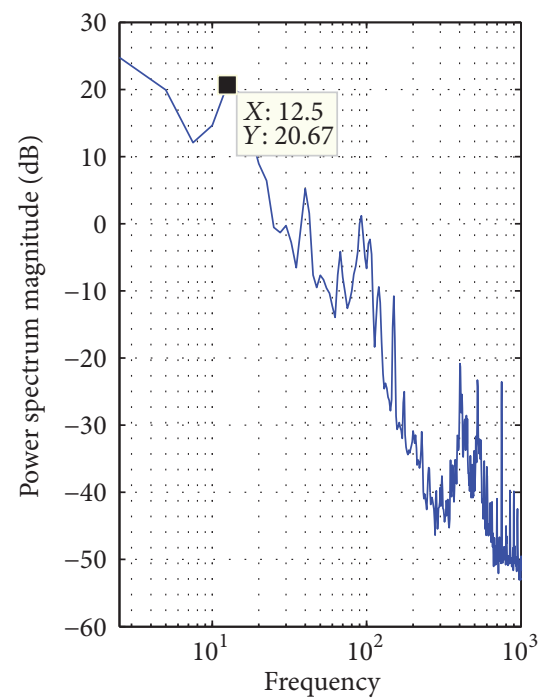

(b)

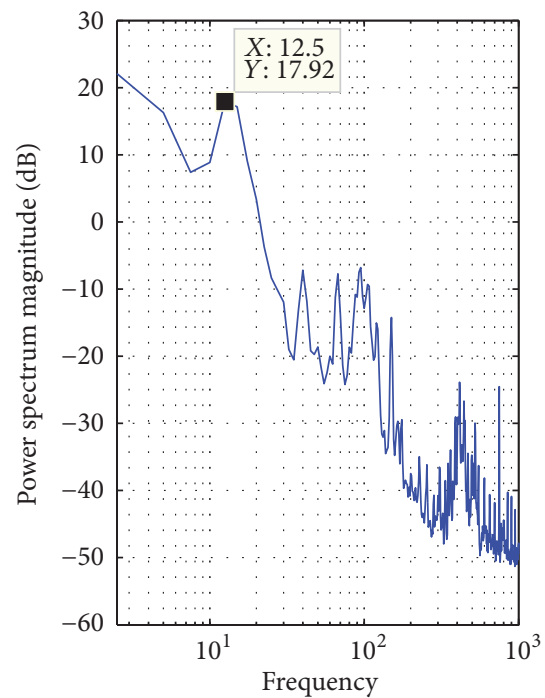

(c)

Figure 13: (a), (b), and (c) are the PSD values of the uncontrolled vibration signal of sensors 1, 2, and 3, respectively.

$$
\begin{aligned}
X_{p} & =0, \\
Y_{p} & =0.05(t-1)-\frac{1}{40 \pi} \sin (2 \pi t), \\
\theta & =0 .
\end{aligned}
$$

The PZT sensor films are bonded onto passive flexible links, as shown in Figure 7; the vibration signal of one PZT sensor film is fed back to two pairs of PZT actuators. Thus, there are three PZT sensors and six pairs of PZT actuators in the whole system. Because the dSPACE controller has a timelag for collecting data, the last 14 seconds of a motion period is chosen as the data collection time. The sampling frequency is set at $10000 \mathrm{~Hz}$ in the MATLAB/Simulation; thus, each sensor collects 140,000 data points.

Figures 13(a), 13(b), and 13(c) are the PSD (Power Spectrum Density) values of the uncontrolled vibration signal of sensors 1, 2, and 3, respectively. As shown in Figure 13, the first-order mode frequency of the system is $12.5 \mathrm{~Hz}$; $40 \mathrm{~Hz}$ and $67.5 \mathrm{~Hz}$ are the second-order and third-order mode frequencies, respectively. The PSD values reflect the energy level of the vibration signal under different mode frequencies. The PSD value of the first-order mode is larger than that of the second-order and third-order mode frequencies; thus, the PSD value of first-order mode frequency can reflect suppression effect of the vibration signal. Modal analysis was implemented for 3-RRR planar parallel robots in [21] based on the modal analysis module of Ansys-Workbench software. The results indicate that the first three-order modes can completely reflect the dynamic characteristics of the system.

Four control schemes are designed in this experiment. Scheme 1 involves three passive flexible links which are controlled at the same time; scheme 2 involves only passive flexible link 1 which is controlled; scheme 3 involves only
TABLE 1: PSD value of the first-order mode frequency.

\begin{tabular}{lccc}
\hline & PSD1 & PSD2 & PSD3 \\
\hline Uncontrolled & 29.58 & 20.67 & 17.92 \\
Scheme 1 & $27.37(-7.47 \%)$ & $14.61(-29.32 \%)$ & $10.34(-42.3 \%)$ \\
Scheme 2 & $27.08(-8.45 \%)$ & $20.65(-0.1 \%)$ & $17.83(-0.5 \%)$ \\
Scheme 3 & $29.6(+0.06 \%)$ & $14.14(-31.59 \%)$ & $17.89(-0.17 \%)$ \\
Scheme 4 & $29.44(-0.47 \%)$ & $20.25(-2.03 \%)$ & $10.16(-43.3 \%)$ \\
\hline
\end{tabular}

Note: PSD1 is the PSD value of the vibration signal of sensor 1 with respect to the first-order mode frequency; PSD2 is the PSD value of the vibration signal of sensor 2 with respect to the first-order mode frequency; PSD3 is the PSD value of the vibration signal of sensor 3 with respect to the first-order mode frequency.

passive flexible link 2 which is controlled; scheme 4 involves only passive flexible link 3 which is controlled.

Figures 14(a), 14(b), and 14(c) are the PSD value of the vibration signal of sensors 1,2 , and 3 with three passive flexible links being controlled at the same time, respectively. Figures 15(a), 15(b), and 15(c) are the PSD value of the vibration signal of sensors 1,2 , and 3 with only passive flexible link 1 being controlled, respectively; Figures 16(a), 16(b), and 16(c) are PSD the value of vibration signal of sensors 1,2 , and 3 with only passive flexible link 2 being controlled, respectively. Figures 17(a), 17(b), and 17(c) are the PSD value of vibration signal of sensors 1,2 , and 3 with only passive flexible link 3 being controlled, respectively.

Table 1 shows the PSD value of three uncontrolled sensors with the above four control schemes regarding the firstorder mode frequency. Without control, the three PSD values are $29.58 \mathrm{~dB}, 20.67 \mathrm{~dB}$, and $17.92 \mathrm{~dB}$. In scheme 1 , the three PSD values are $27.37 \mathrm{~dB}, 14.61 \mathrm{~dB}$, and $10.34 \mathrm{~dB}$; compared with the uncontrolled case, three PSD values descend by 


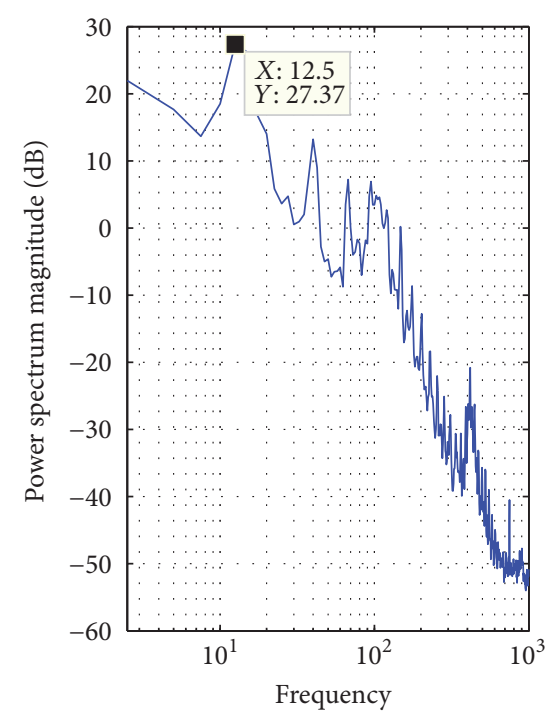

(a)

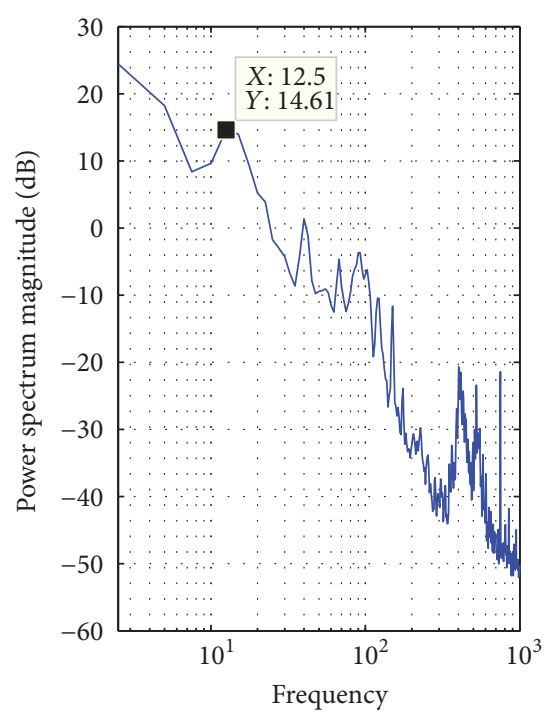

(b)

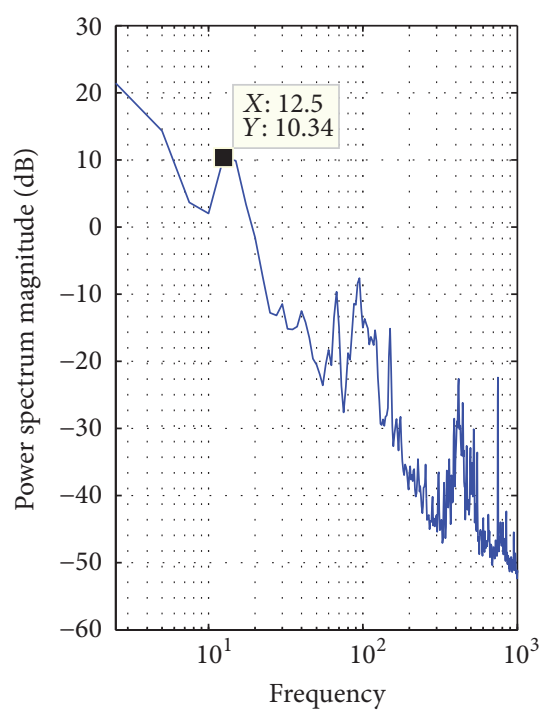

(c)

Figure 14: (a), (b), and (c) are the PSD value of the vibration signal of sensors 1, 2, and 3 with three passive flexible links being controlled at the same time, respectively.

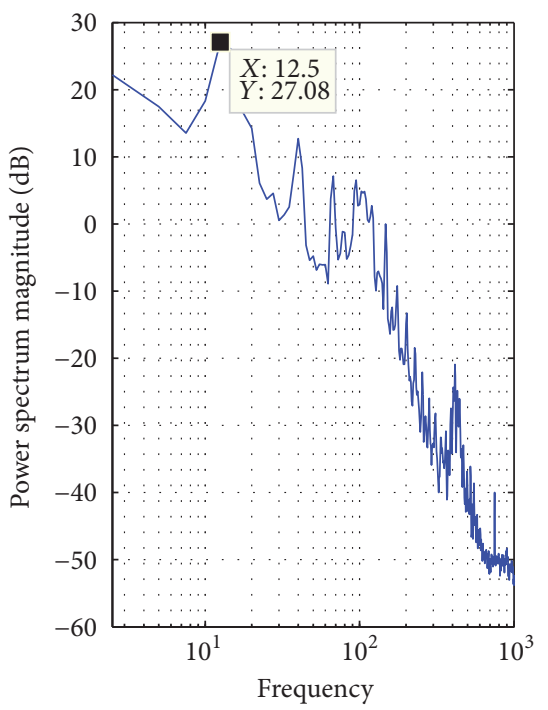

(a)

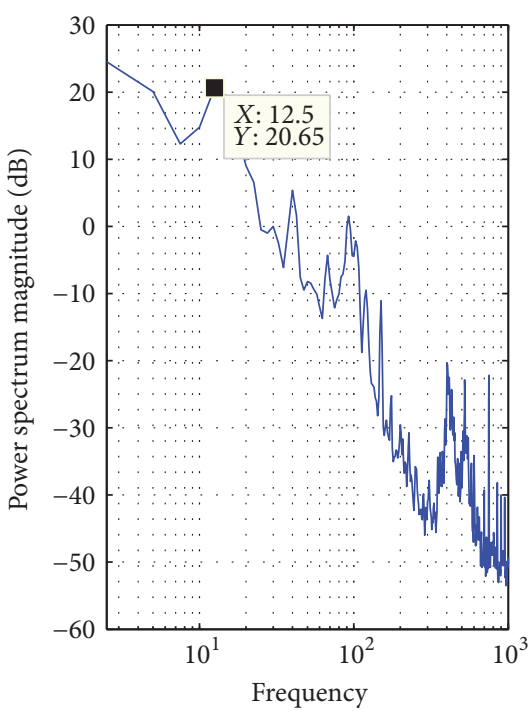

(b)

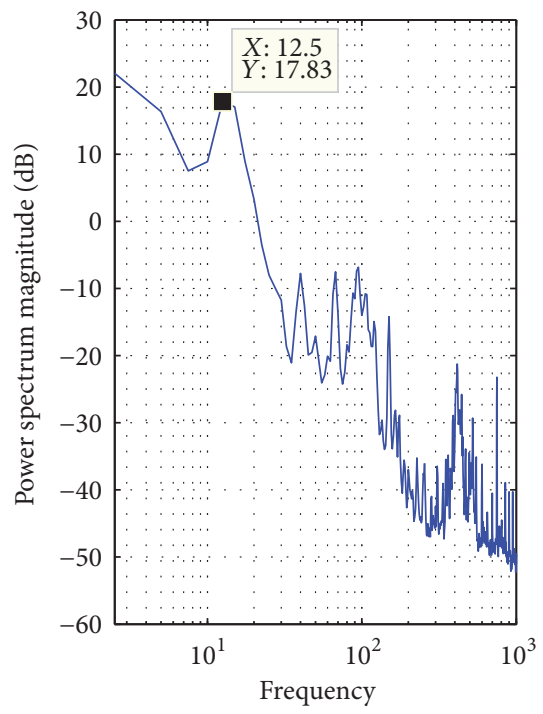

(c)

FIGURE 15: (a), (b), and (c) are PSD value of vibration signal of sensors 1, 2, and 3 with only passive flexible link 1 being controlled, respectively.

$7.47 \%, 29.32 \%$, and $42.3 \%$, respectively. In scheme 2 , the three PSD values are $27.08 \mathrm{~dB}, 20.65 \mathrm{~dB}$, and $17.83 \mathrm{~dB}$; compared with the uncontrolled case, the three PSD values descend by $8.45 \%, 0.1 \%$, and $0.5 \%$. In scheme 3 , three PSD values are $29.6 \mathrm{~dB}, 14.14 \mathrm{~dB}$, and $17.89 \mathrm{~dB}$, respectively; compared with the uncontrolled case, PSD1 increases by $0.06 \%$, PSD2 decreases by $31.59 \%$, and PSD3 decreases by $0.17 \%$. In scheme 4, the three PSD values are $29.6 \mathrm{~dB}, 14.14 \mathrm{~dB}$, and $17.89 \mathrm{~dB}$; compared with the uncontrolled case, the three PSD values descend by $0.47 \%, 2.03 \%$, and $43.3 \%$, respectively.

We can draw the following conclusions from Figures 1317 and Table 1:
(1) Each separate controlled flexible link only suppresses its elastic vibration and cannot suppress the elastic vibration of other flexible links.

(2) The case in which three passive flexible links are controlled at the same time can effectively suppress elastic vibration of all flexible links.

(3) Because coupling between flexible links exists, the control performance of three passive flexible links being controlled at the same time is worse than that of the corresponding separate controlled flexible link. 


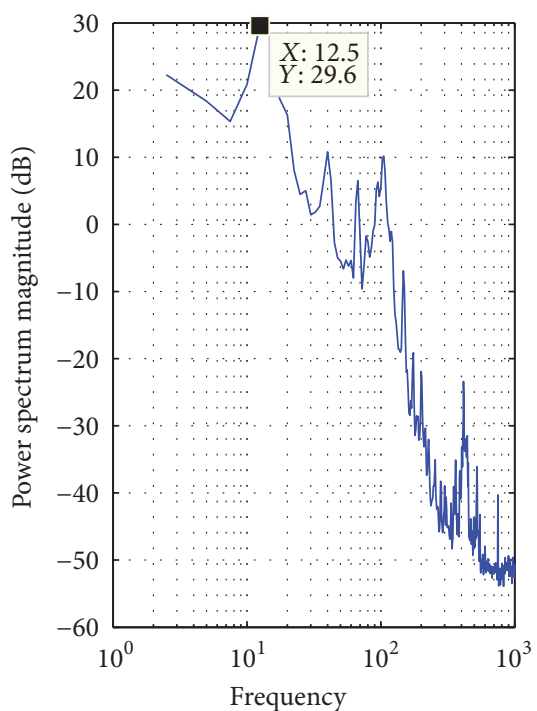

(a)

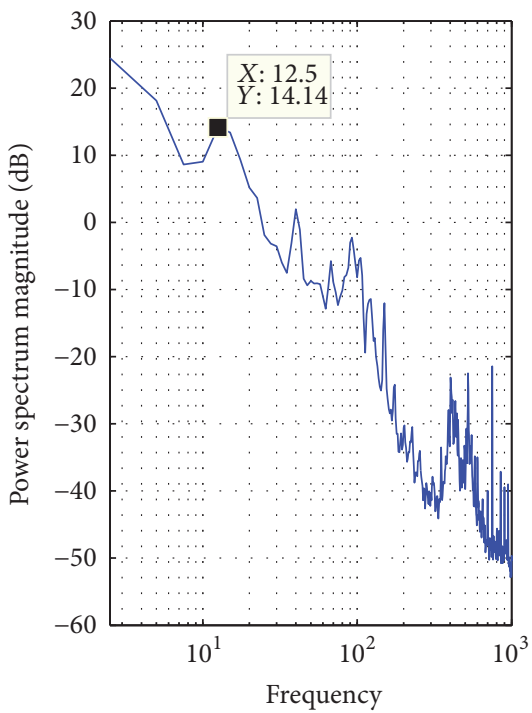

(b)

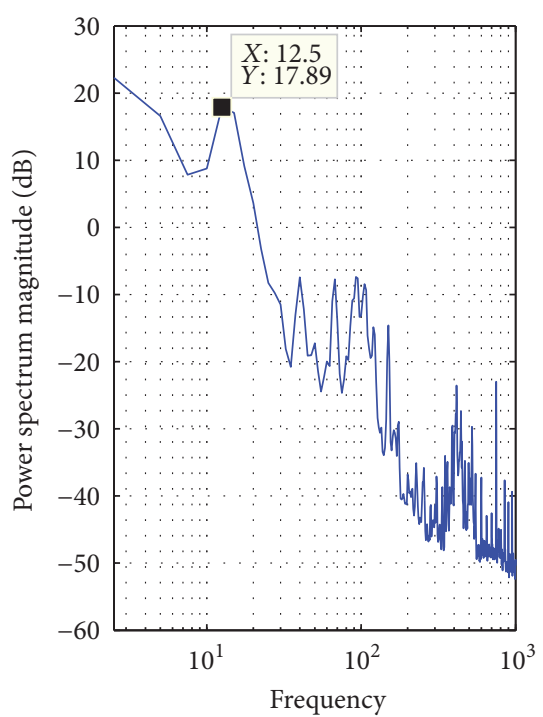

(c)

FIGURE 16: (a), (b), and (c) are PSD value of vibration signal of sensors 1, 2, and 3 with only passive flexible link 2 being controlled, respectively.

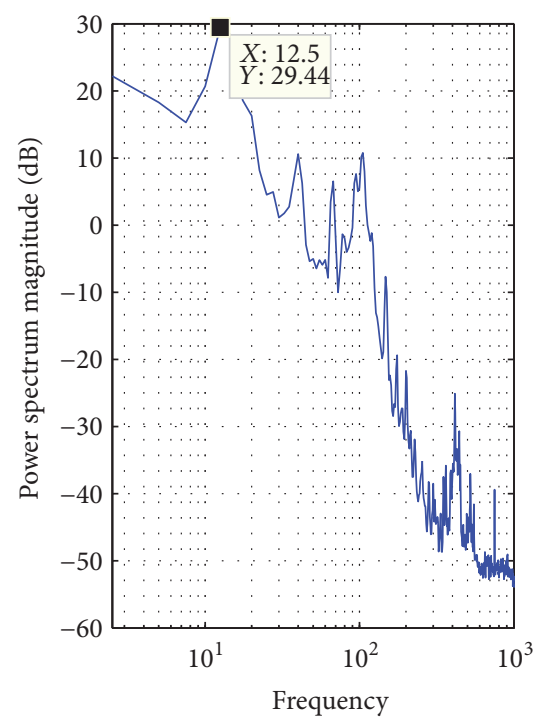

(a)

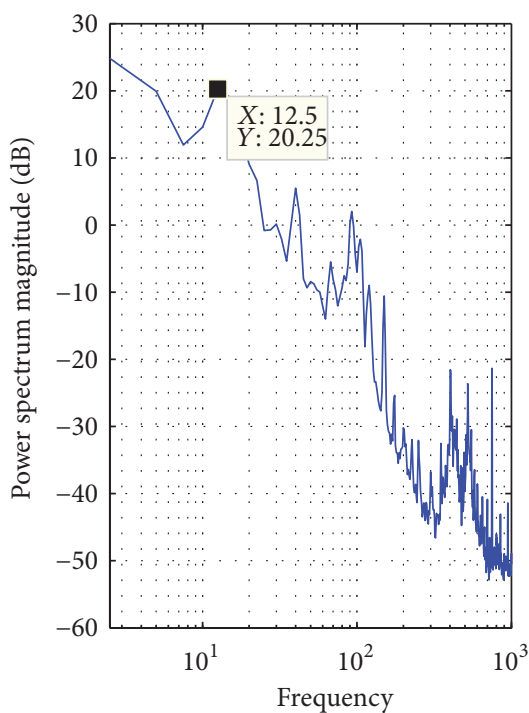

(b)

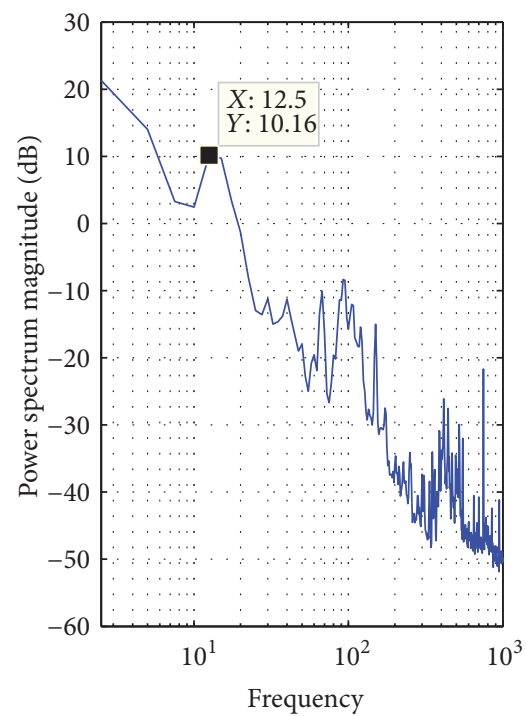

(c)

Figure 17: (a), (b), and (c) are the PSD value of vibration signal of sensors 1, 2, and 3 with only passive flexible link 3 being controlled, respectively.

Figures 18(a), 18(b), 18(c), 18(d), and 18(e) are the vibration signals of passive flexible link 1 for the uncontrolled, scheme 1 , scheme 2, scheme 3, and scheme 4, respectively. As shown in Figure 18, the elastic vibration of the passive flexible link 1 is suppressed in scheme 1 and scheme 2 (see Figures 18(b) and 18(c)), and the other schemes cannot effectively suppress the elastic vibration of passive flexible link 1 (see Figures 18(d) and 18(e)).

Figures 19(a), 19(b), 19(c), 19(d), and 19(e) are the vibration signals of passive flexible link 2 for the uncontrolled, scheme 1 , scheme 2 , scheme 3 , and scheme 4 , respectively. As shown in Figure 19, the elastic vibration of the passive flexible link 2 is suppressed in scheme 1 and scheme 3 (see Figures 19(b) and 19(d)), and the other schemes cannot effectively suppress the elastic vibration of passive flexible link 2 (see Figures 19(c) and 19(e)).

Figures 20(a), 20(b), 20(c), 20(d), and 20(e) are the vibration signals of passive flexible link 3 for the uncontrolled, scheme 1 , scheme 2 , scheme 3 , and scheme 4 , respectively. As shown in Figure 20, the elastic vibration of the passive flexible link 3 is suppressed in scheme 1 and scheme 4 (see Figures 20 (b) and 20(e)), and the other schemes cannot effectively suppress elastic vibration of passive flexible link 2 (see in Figures 20(c) and 20(d)). 


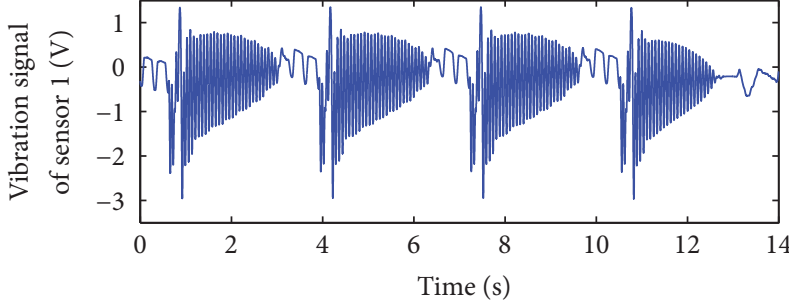

(a)

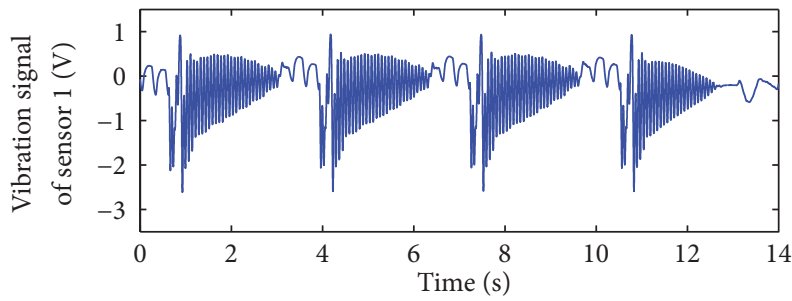

(c)

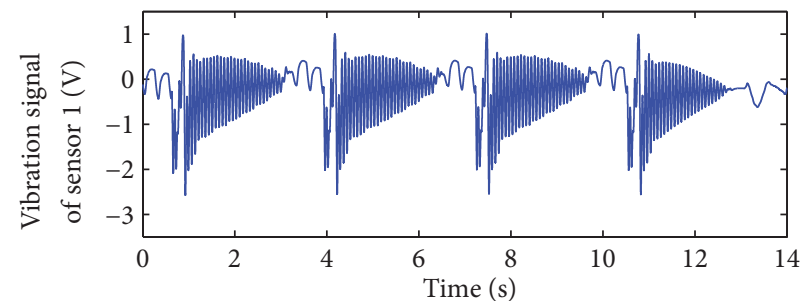

(b)

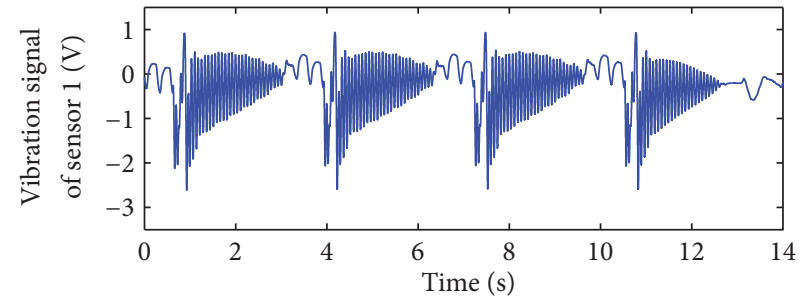

(d)

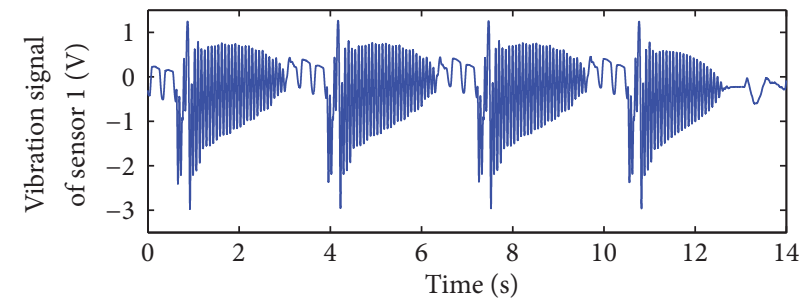

(e)

FIGURE 18: (a), (b), (c), (d), and (e) are the vibration signals of passive flexible link 1 for the uncontrolled, scheme 1 , scheme 2 , scheme 3 , and scheme 4 , respectively.

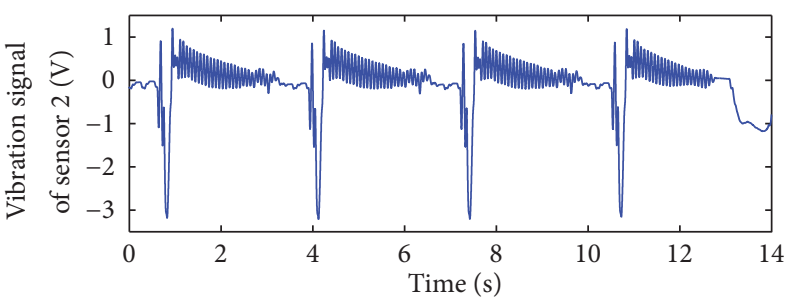

(a)

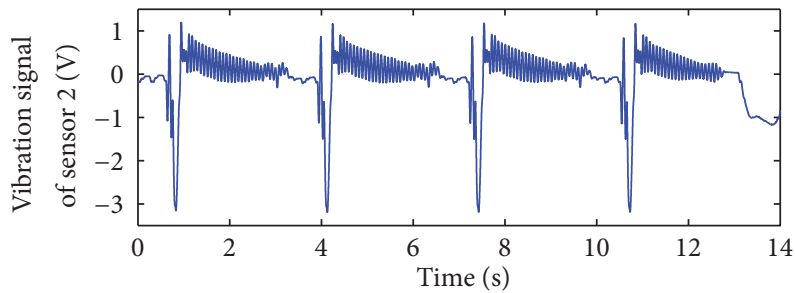

(c)

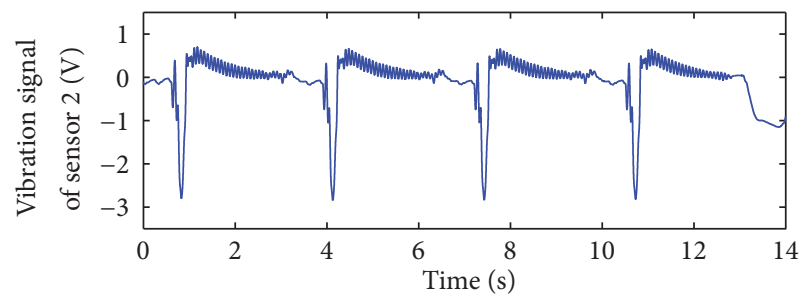

(b)

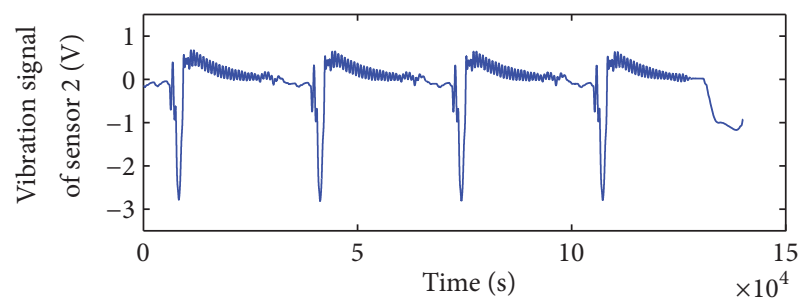

(d)

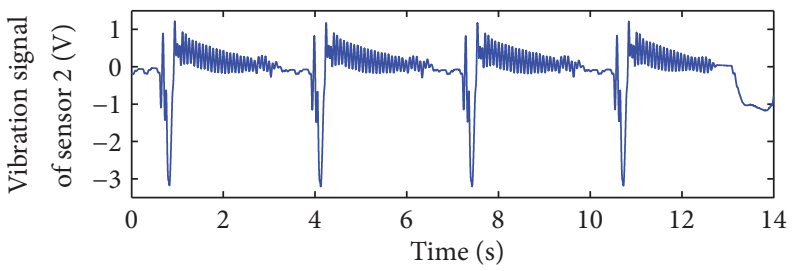

(e)

Figure 19: (a), (b), (c), (d), and (e) are the vibration signals of passive flexible link 2 for the uncontrolled, scheme 1, scheme 2, scheme 3 , and scheme 4 , respectively. 


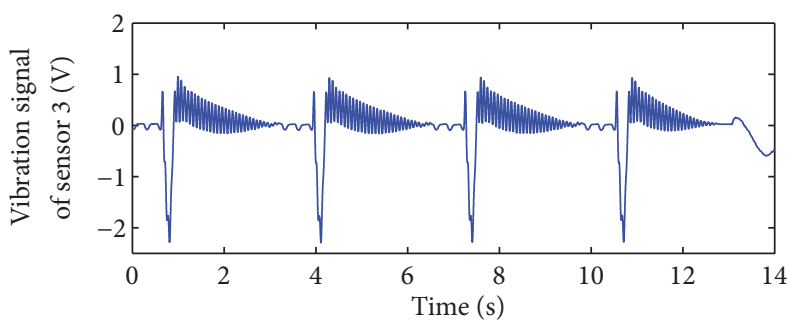

(a)

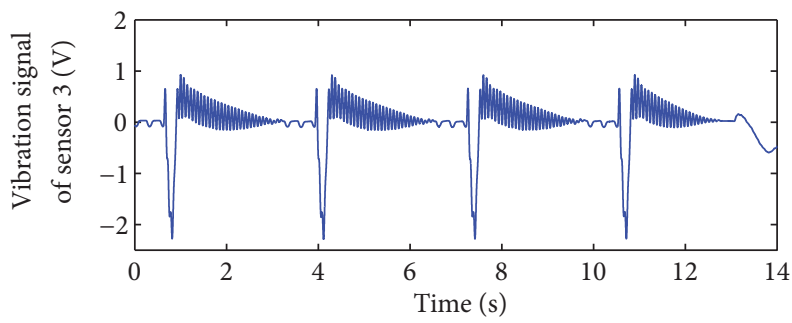

(c)

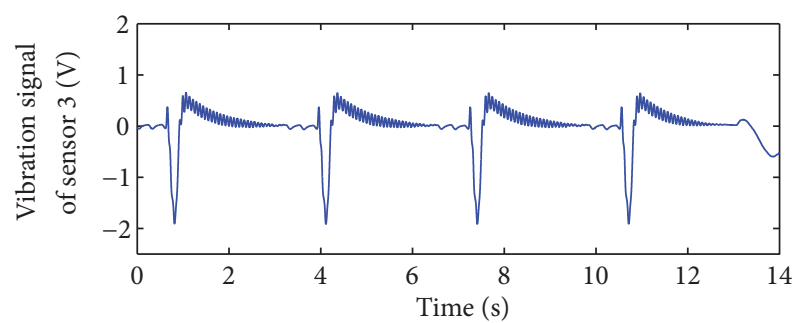

(b)

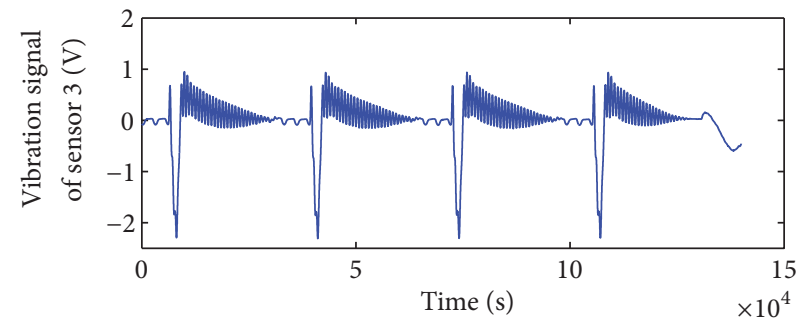

(d)

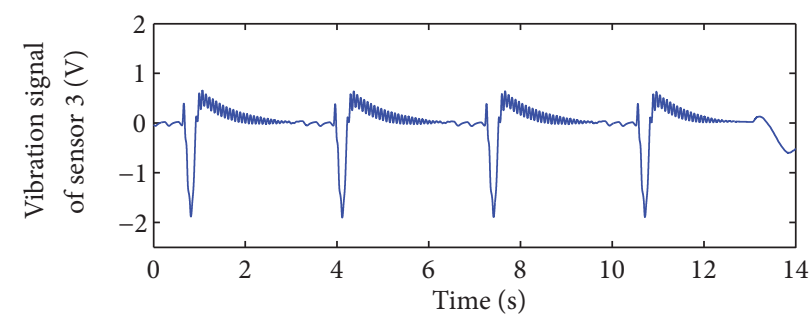

(e)

Figure 20: (a), (b), (c), (d), and (e) are the vibration signals of passive flexible link 3 for the uncontrolled, scheme 1, scheme 2, scheme 3, and scheme 4 , respectively.

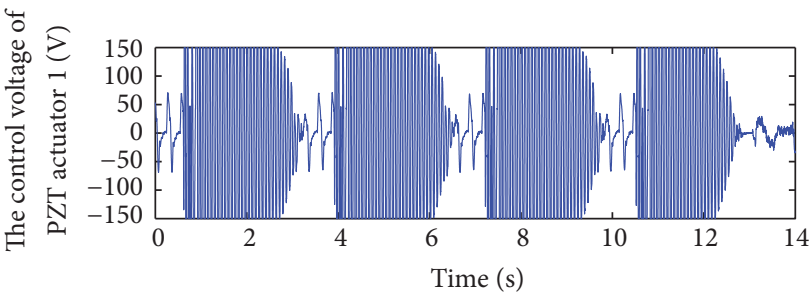

(a)

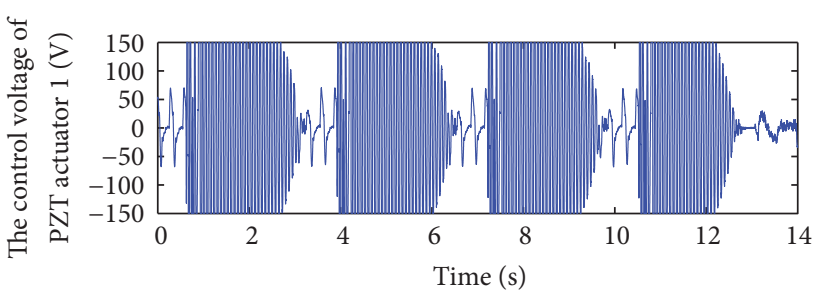

(b)

FIGURE 21: (a) and (b) are the control voltages of PZT actuator 1 with scheme 1 and scheme 2, respectively.

From Figures 18-20, one can find that each separate controlled flexible link can only suppress the elastic vibration of itself and cannot suppress the elastic vibration of the other two flexible links. When three passive flexible links exert control simultaneously, all of the elastic vibrations of three passive flexible links can be suppressed. Because coupling of the elastic vibration between three passive flexible links exists, the control effect is slightly worse than that of each separate controlled flexible link controlling its own vibration; however, the whole control effect of scheme 1 is better than that of the other schemes. Meanwhile, the four control schemes can not only suppress residual elastic vibration of the corresponding passive flexible link but also suppress the elastic vibration of flexible links during the moving of the system.

Figures 21(a) and 21(b) are the control voltages of PZT actuator 1 with scheme 1 and scheme 2, respectively. Figures 22(a) and 22(b) are the control voltages of PZT actuator 2 with scheme 1 and scheme 2, respectively. Figures 23(a) and 23(b) are the control voltages of PZT actuator 3 with scheme 1 and scheme 3 , respectively. Figures 24(a) and 24(b) are the control voltages of PZT actuator 4 with scheme 1 and scheme 3, respectively. Figures 25(a) and 25(b) are the control voltages of PZT actuator 5 with scheme 1 and scheme 4, respectively. Figures 26(a) and 26(b) are 


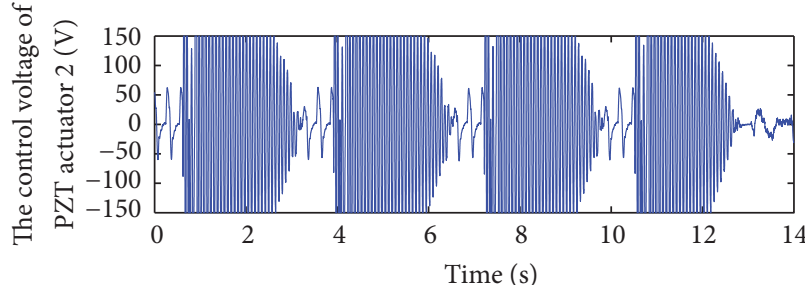

(a)

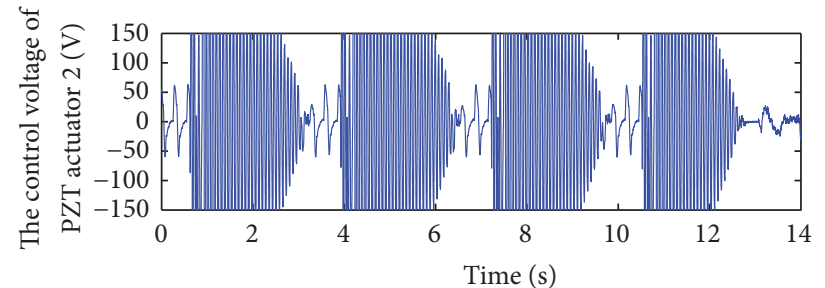

(b)

FIGURE 22: (a) and (b) are the control voltages of PZT actuator 2 with scheme 1 and scheme 2, respectively.

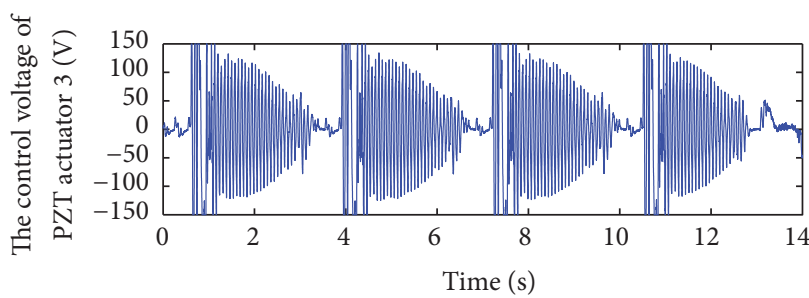

(a)

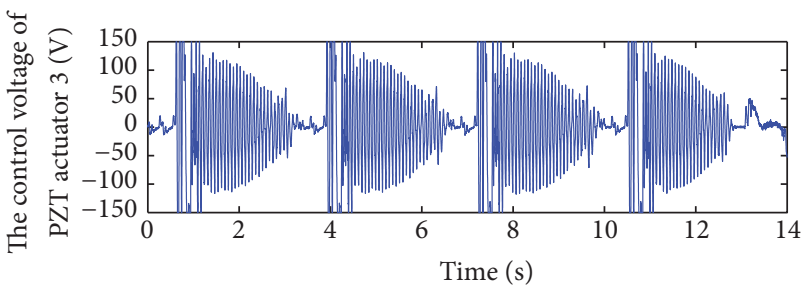

(b)

FIGURE 23: (a) and (b) are the control voltages of PZT actuator 3 with scheme 1 and scheme 3, respectively.

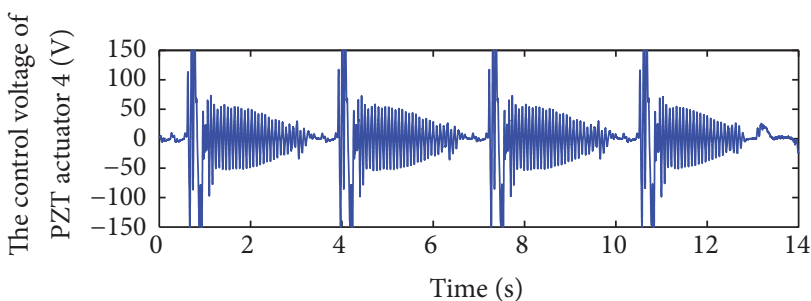

(a)

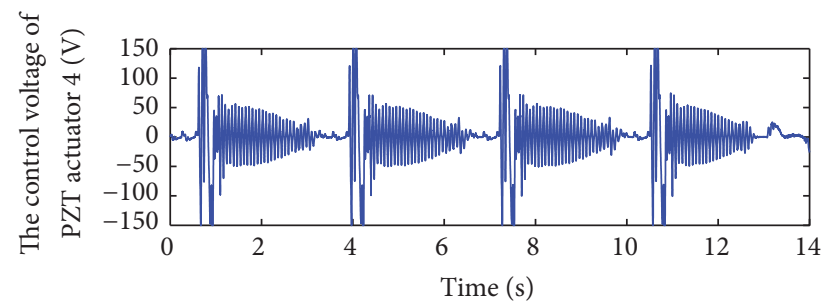

(b)

FIGURE 24: (a) and (b) are the control voltages of PZT actuator 4 with scheme 1 and scheme 3, respectively.

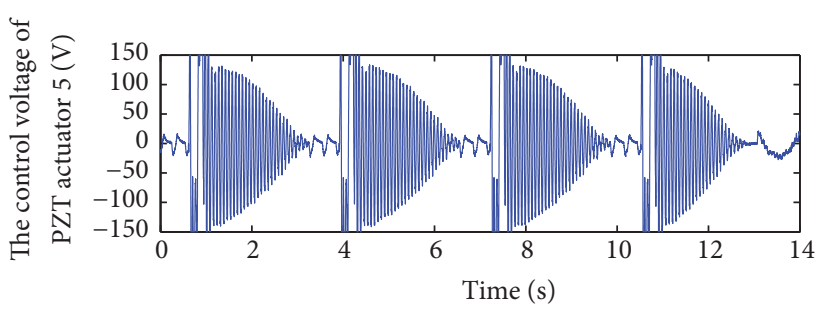

(a)

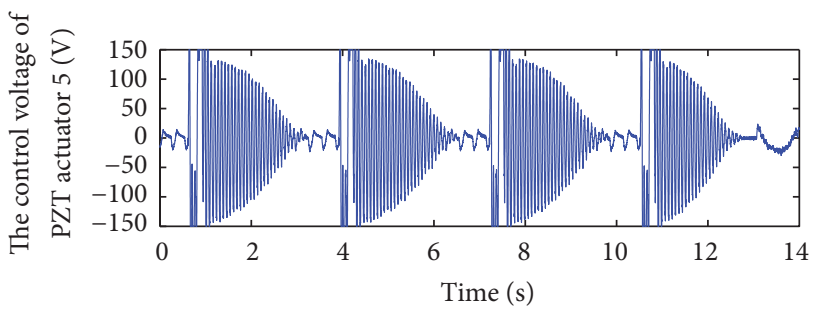

(b)

FIGURE 25: (a) and (b) are the control voltages of PZT actuator 5 with scheme 1 and scheme 4, respectively.

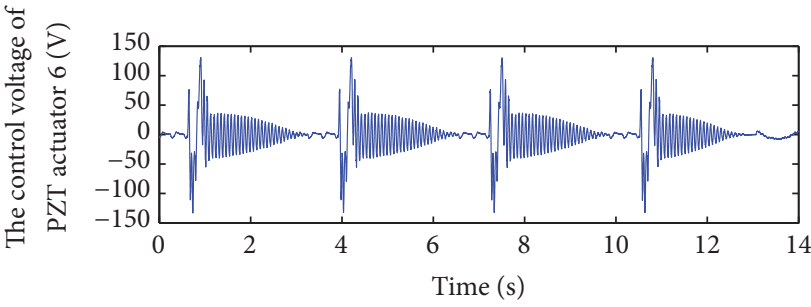

(a)

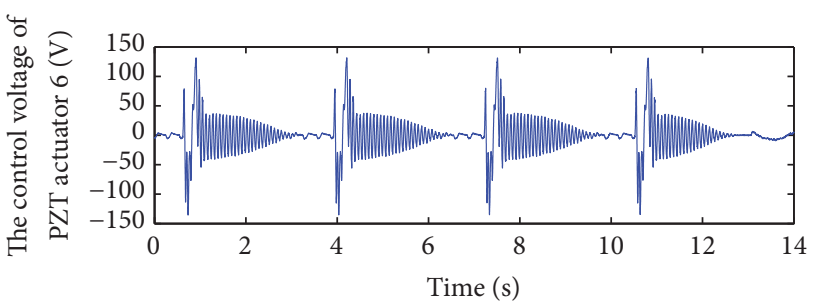

(b)

FIGURE 26: (a) and (b) are the control voltages of PZT actuator 6 with scheme 1 and scheme 4, respectively. 
the control voltages of PZT actuator 6 with scheme 1 and scheme 4 , respectively.

From Figures 21-26, one can observe that the control voltages are consistent for suppressing the vibration of the same passive flexible link. Because the output voltage range of the PZT drive power is limited at $\pm 150 \mathrm{~V}$, when the feedback voltages of DAC of dSPACE go beyond $\pm 10 \mathrm{~V}$, the output voltages of the PZT drive power will be intercepted to $\pm 150 \mathrm{~V}$ (feedback voltages are amplified 15 times). From Figures 2126 and Table 1, one can observe that although the control voltage exerted on PZT actuator of passive flexible link 1 is very high (peak value), the control effect is not adequate, with the maximum decrease of PSD1 of $8.45 \%$. The control voltages exerted on passive flexible links 2 and 3 are smaller than the control voltage of passive flexible link 1, but the control effect is better than passive flexible link 1 (passive flexible link 2 decreases by 31.59\%; passive flexible link 3 decreases by $43.3 \%$ ) because the control effect is closely related to the pose of the planar 3-RRR parallel robots and the motion trajectory of the moving platform. In general, due to the usage of mode filter technology, a time-lag exists between the control signal and the original signal. Thus, the control effect is not completely satisfying.

\section{Conclusions}

An active vibration control experiment of plane 3-RRR flexible parallel robots was studied in this paper. The strain rate feedback controller was designed based on the general motion equation, which considers the direct and inverse piezoelectric equation of the PZT actuator and the PZT sensor. Four control schemes are designed in the experiment: three passive flexible links are controlled at the same time: only passive flexible link 1 is controlled, only passive flexible link 2 is controlled, and only passive flexible link 3 is controlled. The experimental results indicate that each separate controlled flexible link only suppresses its own elastic vibration and cannot suppress the elastic vibration of the other flexible links, whereas three passive flexible links being controlled at the same time can effectively suppress the elastic vibration of all of the flexible links. The manufacture error and assembly error of the experiment platform, friction, time-lag, and gap between joints lead to the gain parameter not completely matching the theoretical value. The gain parameter must be optimized to improve the control performance. There are many highfrequency noise signals that come from the servomotor, and the rigid motion and flexibility of the joints will mix into the collected signals through the PZT sensor. Filter technology is used to remove high-frequency noise signals, but phase-lag exists in the filtered signal that influences the control performance. Thus, the performance of the strain rate feedback controller will be confined, and these issues will be considered to improve the controller performance in a future study.

\section{Competing Interests}

The authors declare that they have no competing interests.

\section{Acknowledgments}

This work was supported by the National Natural Science Foundation of China (Grants nos. U1501247, 61603103, and 51505092), the of Natural Science Foundation Guangdong Province (Grants nos. 2014A030313616, 2015A030310181, and 2016A030310293), the Science and Technology Planning Project of Guangdong Province (2014A010104017 and 2015B010101015), and the Science and Technology Innovation Project of Foshan (2015AG10018). These supports are greatly acknowledged.

\section{References}

[1] Z. Xianmin, S. Changjian, and A. G. Erdman, "Active vibration controller design and comparison study of flexible linkage mechanism systems," Mechanism and Machine Theory, vol. 37, no. 9, pp. 985-997, 2002.

[2] I. Chopra, "Review of state of art of smart structures and integrated systems," AIAA Journal, vol. 40, no. 11, pp. 2145-2187, 2002.

[3] R. Alkhatib and M. F. Golnaraghi, "Active structural vibration control: a review," The Shock and Vibration Digest, vol. 35, no. 5, pp. 367-383, 2003.

[4] D. J. Zhang, Y. W. Liu, and R. L. Houston, "On dynamic stiffening of flexible bodies having high angular velocity," Mechanics of Structures and Machines, vol. 24, no. 3, pp. 313-329, 1996.

[5] R. B. He, S. J. Zheng, and H. T. Wang, "Independent modal variable structure fuzzy active vibration control of cylindrical thin shells laminated with photostrictive actuators," Shock and Vibration, vol. 20, no. 4, pp. 693-709, 2013.

[6] M. Benosman and G. Le Vey, "Control of flexible manipulators: a survey," Robotica, vol. 22, no. 5, pp. 533-545, 2004.

[7] S. K. Dwivedy and P. Eberhard, "Dynamic analysis of flexible manipulators, a literature review," Mechanism and Machine Theory, vol. 41, no. 7, pp. 749-777, 2006.

[8] T. Wasfy and A. Noor, "Computational strategies for flexible multibody systems," Mechanism and Machine Theory, vol. 56, no. 6, pp. 553-614, 2003.

[9] H. N. Rahimi and M. Nazemizadeh, "Dynamic analysis and intelligent control techniques for flexible manipulators: a review," Advanced Robotics, vol. 28, no. 2, pp. 63-76, 2014.

[10] K.-H. Rew, J.-H. Han, and I. Lee, "Multi-modal vibration control using adaptive positive position feedback," Journal of Intelligent Material Systems and Structures, vol. 13, no. 1, pp. 1322, 2002.

[11] S. O. R. Moheimani, B. J. G. Vautier, and B. Bhikkaji, "Experimental implementation of extended multivariable PPF control on an active structure," IEEE Transactions on Control Systems Technology, vol. 14, no. 3, pp. 443-455, 2006.

[12] Z.-C. Qiu, J.-D. Han, X.-M. Zhang, Y.-C. Wang, and Z.-W. Wu, "Active vibration control of a flexible beam using a noncollocated acceleration sensor and piezoelectric patch actuator," Journal of Sound and Vibration, vol. 326, no. 3-5, pp. 438-455, 2009.

[13] J. Orivuori and K. Zenger, "Comparison and performance analysis of some active vibration control algorithms," Journal of Vibration and Control, vol. 20, no. 1, pp. 94-130, 2014. 
[14] M. Preiswerk and A. Venkatesh, "An analysis of vibration control using piezoceramics in planar flexible-linkage mechanisms," Journal of Smart Materials and Structures, vol. 3, no. 2, pp. 190-200, 1994.

[15] J. Shan, H.-T. Liu, and D. Sun, "Slewing and vibration control of a single-link flexible manipulator by positive position feedback (PPF)," Mechatronics, vol. 15, no. 4, pp. 487-503, 2005.

[16] X. P. Zhang, J. K. Mills, and W. L. Cleghorn, "Experimental implementation on vibration mode control of a moving 3-PRR flexible parallel manipulator with multiple PZT transducers," Journal of Vibration and Control, vol. 16, no. 13, pp. 2035-2054, 2010.

[17] Q. Zhang, J. Jin, J. Zhang, and C. Zhao, "Active vibration suppression of a 3-DOF flexible parallel manipulator using efficient modal control," Shock and Vibration, vol. 2014, Article ID 953694, 10 pages, 2014.

[18] Q. H. Zhang and X. M. Zhang, "Dynamic analysis of planar 3-RRR flexible parallel robots under uniform temperature change," Journal of Vibration and Control, vol. 21, no. 1, pp. 81104, 2015.

[19] Q. H. Zhang, X. R. Fang, and X. M. Zhang, "Dyanmic analysis of planar 3-RRR flexible parallel robots with dynamic stiffening," Shock and Vibration, vol. 2014, Article ID 370145, 13 pages, 2014.

[20] Q. H. Zhang and X. M. Zhang, "Active residual vibration control of planar 3-RRR flexible parallel robots," Transactions of the Chinese Society for Agricultural Machinery, vol. 44, no. 2, pp. 232-266, 2013.

[21] Q. H. Zhang, X. M. Zhang, and J. L. Liang, "Dynamic analysis of planar 3-RRR flexible parallel robot," in Proceedings of the IEEE International Conference on Robotics and Biomimetics (ROBIO '12), pp. 154-159, Guanghzhou, China, December 2012.

[22] X. P. Zhang, Dynamic modeling and active vibration control of a planar 3-PRR parallel manipulator with three flexible links [Ph.D. thesis], University of Toronto, Toronto, Canada, 2009.

[23] X. Zhang, C. Shao, Y. Shen, and A. G. Erdman, "Complex mode dynamic analysis of flexible mechanism systems with piezoelectric sensors and actuators," Multibody System Dynamics, vol. 8, no. 1, pp. 51-70, 2002.

[24] J. M. Mayo, D. García-Vallejo, and J. Domínguez, "Study of the geometric stiffening effect: comparison of different formulations," Multibody System Dynamics, vol. 11, no. 4, pp. 321-341, 2004.

[25] J. F. Hu, Dynamic modeling and active vibration control of a planar 3-PRR parallel manipulator with three flexible links [Ph.D. thesis], South China University of Technology, Guangzhou, China, 2010. 


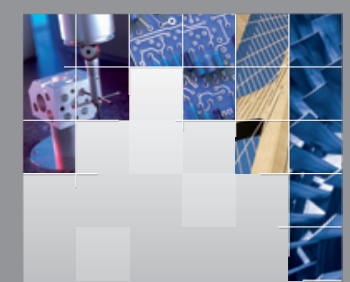

\section{Enfincering}
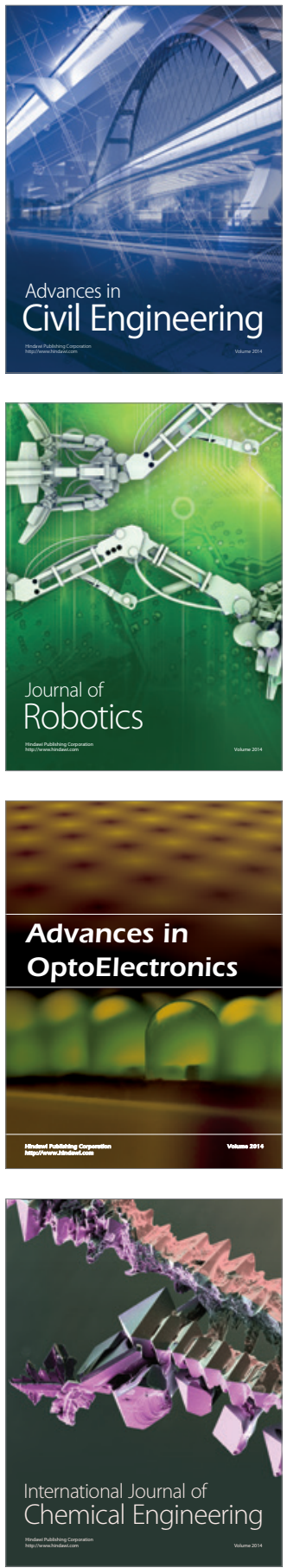

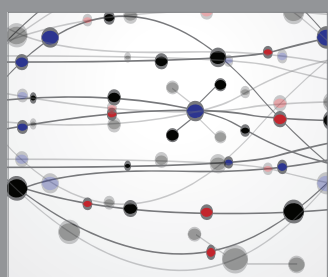

The Scientific World Journal

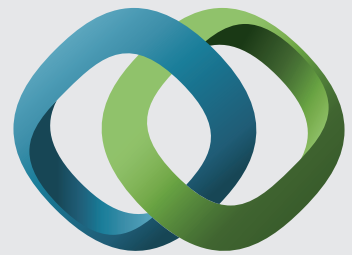

\section{Hindawi}

Submit your manuscripts at

http://www.hindawi.com
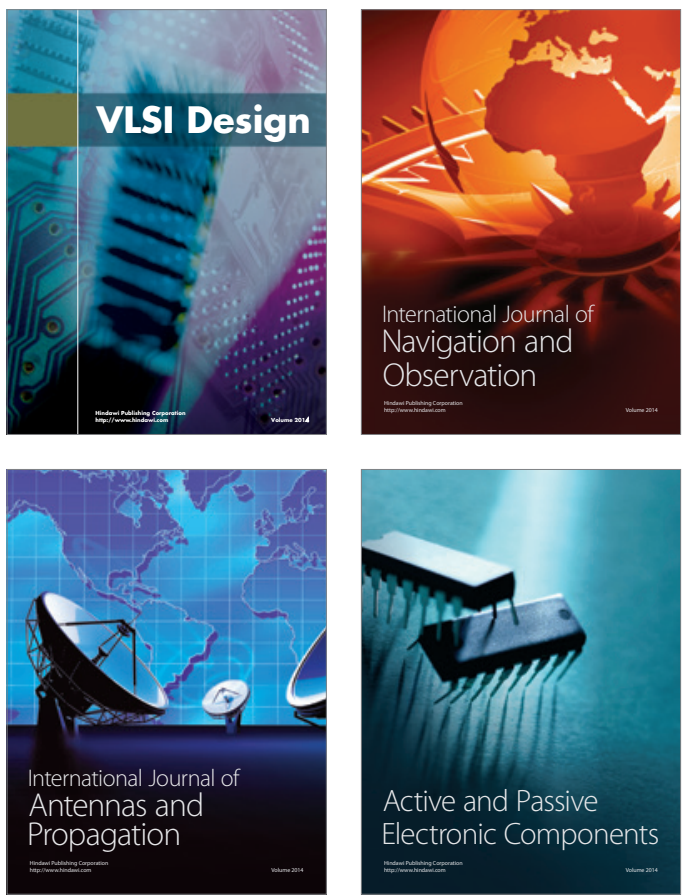
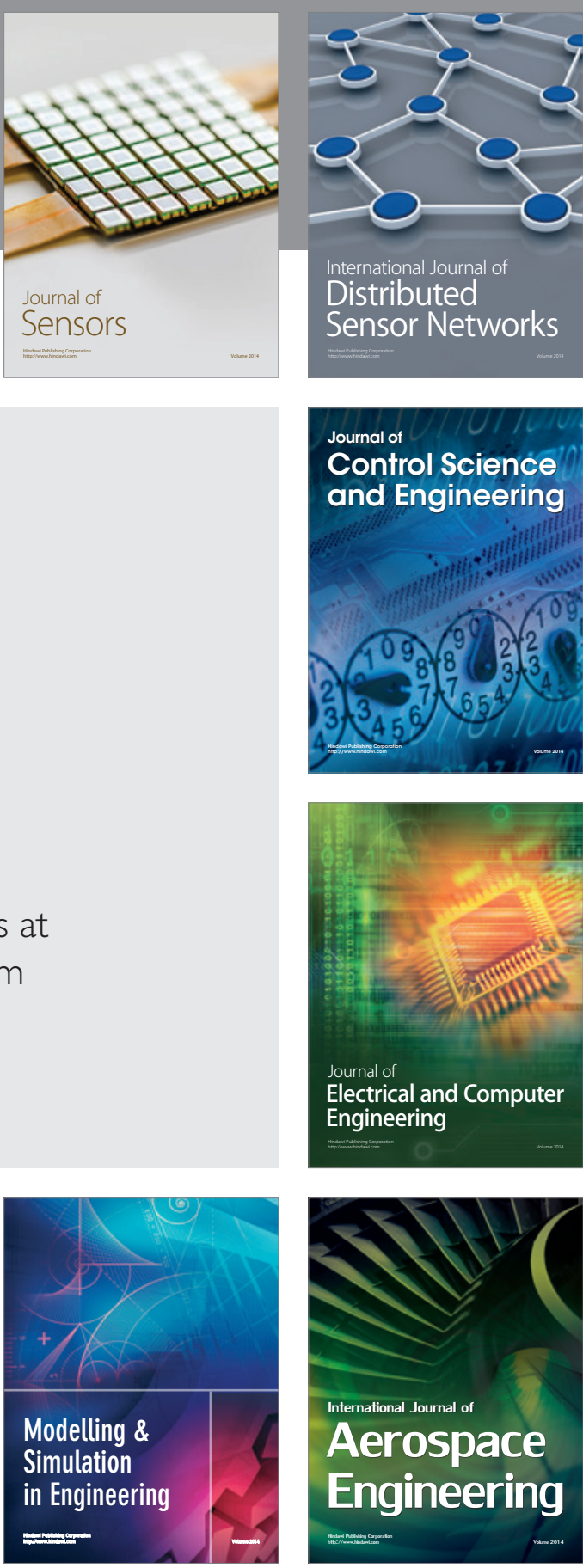

International Journal of

Distributed

Sensor Networks

Journal of

Control Science

and Engineering
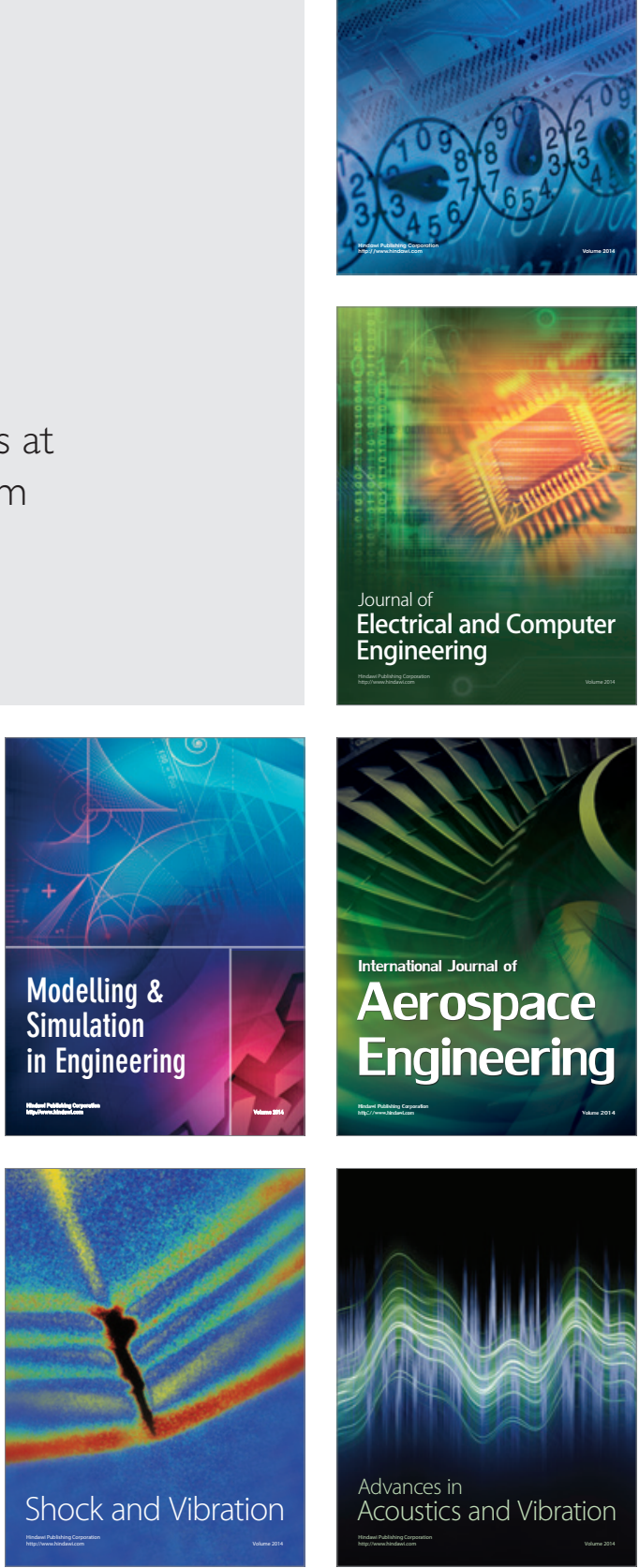